\title{
Multi-degree-of-freedom systems with a Coulomb friction contact: analytical boundaries of motion regimes
}

\author{
Luca Marino • Alice Cicirello
}

Received: 1 July 2020 / Accepted: 12 January 2021 / Published online: 2 March 2021

(C) The Author(s) 2021, corrected publication 2021

\begin{abstract}
This paper proposes an approach for the determination of the analytical boundaries of continuous, stick-slip and no motion regimes for the steady-state response of a multi-degree-of-freedom (MDOF) system with a single Coulomb contact to harmonic excitation. While these boundaries have been previously investigated for single-degree-of-freedom (SDOF) systems, they are mostly unexplored for MDOF systems. Closed-form expressions of the boundaries of motion regimes are derived and validated numerically for two-degree-of-freedom (2DOF) systems. Different configurations are observed by changing the mass in contact and by connecting the rubbing wall to: (i) the ground, (ii) the base or (iii) the other mass. A procedure for extending these results to systems with more than 2DOFs is also proposed for (i)-(ii) and validated numerically in the case of a 5DOF system with a ground-fixed contact. The boundary between continuous and stick-slip regimes is obtained as an extension of Den Hartog's formulation for SDOF systems with Coulomb damping (Trans Am Soc Mech Eng 53: 107-115, 1931). The boundary between motion and
\end{abstract}

L. Marino $(\varangle) \cdot$ A. Cicirello

Department of Engineering Science, University of Oxford, Parks Road, Oxford OX1 3PJ, UK

e-mail: luca.marino@eng.ox.ac.uk

A. Cicirello

Department of Engineering Structures, Faculty of Civil

Engineering and Geosciences, Delft University of

Technology, Stevinweg 1, Delft 2628, Netherlands

e-mail: a.cicirello@tudelft.nl no motion regimes is derived with an ad hoc procedure, based on the comparison between the overall dynamic load and the friction force acting on the mass in contact. The boundaries are finally represented in a twodimensional parameter space, showing that the shape and the extension of the regions associated with the three motion regimes can change significantly when different physical parameters and contact configurations are considered.

Keywords Coulomb damping · Friction · Multidegree of freedom - Stick-slip · Motion regimes · Base motion $\cdot$ Joined base-wall motion

\section{Introduction}

Improving the fundamental knowledge of the dynamic behaviour of friction damped systems is one of the most pressing challenges in structural dynamics. In fact, friction joints and interfaces are found in a wide range of mechanical and civil structures. Furthermore, friction dampers are often introduced in engineering applications to achieve energy dissipation, isolation and vibration control. However, their effect on the dynamic performances of such systems is not yet fully understood.

The dynamic response of systems with frictional interfaces is not always continuous. In fact, the following behaviours can also be observed in the relative motion between the surfaces of the joint: (i) stops can periodically occur in the motion, leading to the 
so-called stick-slip regime; (ii) the surfaces in contact can be completely stuck, a condition which will be referred to as no motion regime. These phenomena can have undesired and critical consequences on engineering structures if not accounted for during the design stage. For example, stick-slip can result in noise, energy loss, excessive wear and component failures [1], while unexpected full-stuck conditions in friction contacts can lead to a significant reduction of damping effects and alter the dynamic behaviour of the structure.

The goal of this paper is the development of an analytical approach for the formulation of the boundaries of these motion regimes for multi-degree-of-freedom (MDOF) systems with a Coulomb friction contact. Specifically, two different boundaries will be investigated: (i) between continuous and stick-slip regimes; (ii) between motion and no motion regimes. Different contact configurations will be explored, considering different masses involved in the friction contact and either fixed or oscillating wall cases. The boundaries will be represented in two-dimensional parameter spaces, which will be therefore divided into three regions associated with continuous, stick-slip and no motion regimes. The observation of these parameter spaces will enable the determination of the motion regime for each given set of parameters of the system, of the contact and of the excitation considered.

The steady-state response features of harmonically excited systems presenting a Coulomb contact between the mass and a fixed wall were widely explored in the literature (see, e.g. [2-7]) for the single-degree-offreedom (SDOF) case. Specifically, the determination of an upper bound for continuous non-sticking motion was mainly tackled by Den Hartog [2] and Hong and Liu [5]; in addition, many authors [7-9] further investigated the motion bounds accounting also for the different number of stops per cycle in stick-slip regime. In these systems, the upper bound for the presence of mass motion, either in continuous or stick-slip regime, is obtained when the amplitudes of the exciting and friction forces are equal, independently of the exciting frequency. However, a different behaviour was observed by Marino et al. [10] in Coulomb damped SDOF systems subject to joined base-wall harmonic excitation, where the rubbing wall is assumed to oscillate jointly with the base. The wall motion introduces a different dynamic load on the mass, whose amplitude becomes proportional to the square of the exciting frequency.
Therefore, also the upper bound for the presence of a relative motion in the contact will become frequencydependent.

The response of MDOF systems to harmonic excitation is often investigated numerically [11-13]. As time integration can be computationally expensive [14], frequency domain methods such as harmonic balance [15-18] or multi-harmonic analysis [19-21] have been explored. A more complete review on friction damped systems and current numerical approaches can be found in reference [22]. Analytical approaches are also described in the literature for 2DOF systems with a Coulomb contact: in 1966, Yeh [23] derived a closedform solution for the continuous non-sticking response of 2DOF systems with combined viscous and Coulomb damping, while more recently further theoretical developments were presented in references [24-26]. Alternative approaches such as the method of averaging have also been explored for finding approximate solutions when the number of DOFs of the system is larger [27]. Finally, the problem has often been addressed by introducing an equivalent viscous damper to account for the energy loss due to the frictional dissipation [14,28,29]. Nevertheless, to the best of the authors' knowledge, the problem of the determination of the boundaries among continuous, stick-slip and no motion regimes has never been tackled for these systems.

In this contribution, the upper bound for nonsticking motion is evaluated by extending Den Hartog's approach [2]. In fact, Den Hartog determined the continuous dynamic response and the boundary between continuous and stick-slip motion regimes by considering a time interval, equal to half period of motion in steady-state conditions, where the governing equations are linear. This approach can also be used to investigate the behaviour of MDOF systems if a single friction contact, i.e. a single nonlinearity, is considered. In particular, this enables the use of standard modal analysis to evaluate the terms appearing in the boundary equation. An ad hoc procedure is introduced for determining the domain where relative motion is allowed in the friction contact. The approach is based on the evaluation of the overall dynamic load acting on the mass in contact when it is fixed. The upper bound is then described by equating the amplitudes of this dynamic force and of the friction force.

Three different types of friction contacts are investigated for two-degree-of-freedom (2DOF) systems: 
- ground-fixed wall contacts (Sect. 2), achieved between one of the masses and a fixed wall;

- base-fixed wall contacts (Sect. 3), achieved between one of the masses and a wall oscillating jointly with the base;

- mass-fixed wall contacts (Sect. 4), where two masses are connected by a spring and a Coulomb contact in parallel.

These MDOF systems can provide a simplified model for several engineering applications, including friction dampers for civil building, car suspensions, bladed discs and many others. For each of the listed contact configurations, the analytical boundaries are evaluated and validated with results found using a numerical approach, which is introduced in Sect. 4. Subsequently, the analytical results for ground-fixed and base-fixed contacts are extended to systems with more than two DOFs in Sects. 2 and 3, respectively; particularly, a numerical validation is proposed for the case of a $5 \mathrm{DOF}$ system with a ground-fixed contact applied on either the fourth or the second mass at the end of Sect. 2.

\section{Ground-fixed wall contacts}

This section focuses on the study of a MDOF system with a Coulomb contact between one of the masses of the system and a ground-fixed wall. The purpose of this investigation is determining which motion regime (continuous, stick-slip or no motion) can be observed for each set of physical parameters of the problem. Den Hartog's approach for the determination of motion regimes in SDOF systems [2] is recalled and extended to MDOF systems by considering the superposition of modal behaviour. Analytical expressions for the bounds of the different motion regimes are presented and validated with numerical results obtained using the approach described in Sect. 4 for 2DOF systems with a fixed contact on either the lower or upper mass and for a 5DOF system with the either fourth or the second mass in contact to a fixed wall.

\subsection{Governing equations and dimensionless groups definition}

Let us consider a 2DOF system composed of two masses $m_{1}$ and $m_{2}$ and two springs of stiffness $k_{1}$ and $k_{2}$, where either the lower mass (Fig. 1a) or the upper

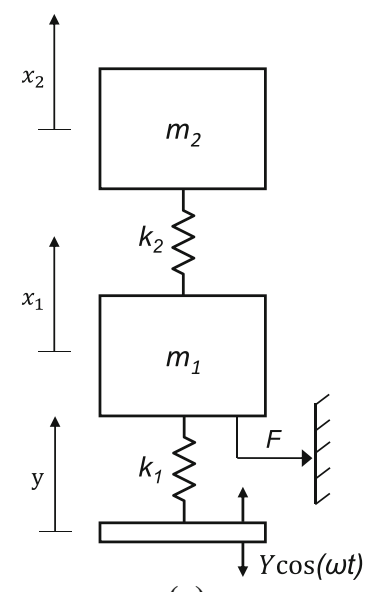

(a)

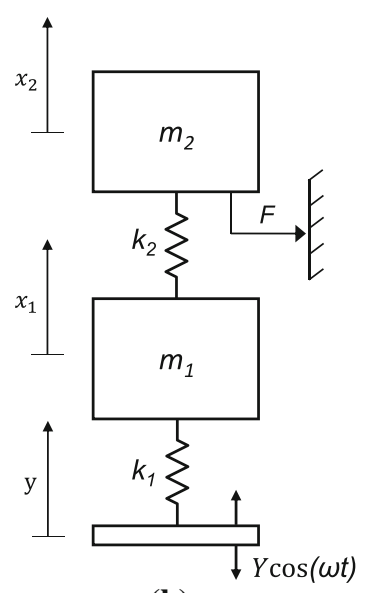

(b)
Fig. 1 2DOF system under harmonic base excitation with a Coulomb ground-fixed wall contact on $\mathbf{a}$ the lower mass or $\mathbf{b}$ the upper mass

mass (Fig. 1b) is rubbing against a ground-fixed wall generating a Coulomb friction force of amplitude $F$. Such systems are excited by a harmonic base motion of amplitude $Y$ and frequency $\omega$, described by the coordinate $y$. The coordinates describing the position of the two masses are $x_{1}$ and $x_{2}$, respectively. The governing equations of each of these systems can be written, respectively, as:

$m_{1} \ddot{x}_{1}+\left(k_{1}+k_{2}\right) x_{1}-k_{2} x_{2}+F \operatorname{sgn}\left(\dot{x}_{1}\right)=k_{1} y$

$m_{2} \ddot{x}_{2}-k_{2} x_{1}+k_{2} x_{2}=0$

and:

$m_{1} \ddot{x}_{1}+\left(k_{1}+k_{2}\right) x_{1}-k_{2} x_{2}=k_{1} y$

$m_{2} \ddot{x}_{2}-k_{2} x_{1}+k_{2} x_{2}+F \operatorname{sgn}\left(\dot{x}_{2}\right)=0$

where $y=Y \cos (\omega t)$ and:

$\operatorname{sgn}\left(\dot{x}_{i}\right)=\left\{\begin{aligned} 1 & \text { if } \dot{x}_{i}>0 \\ {[-1,1] } & \text { if } \dot{x}_{i}=0 \\ -1 & \text { if } \dot{x}_{i}<0\end{aligned}\right.$

When the sliding velocity is zero, the sgn() function is meant to assume any value between -1 and 1 . The actual value will be such that the system is in equilibrium, i.e. the sum of the spring forces and of the friction force is zero. By using the definition in Eq. (3), it is 


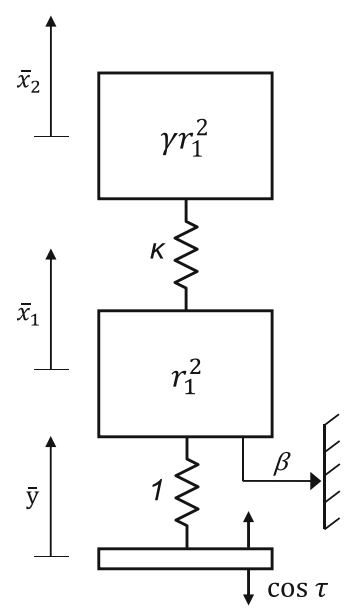

(a)

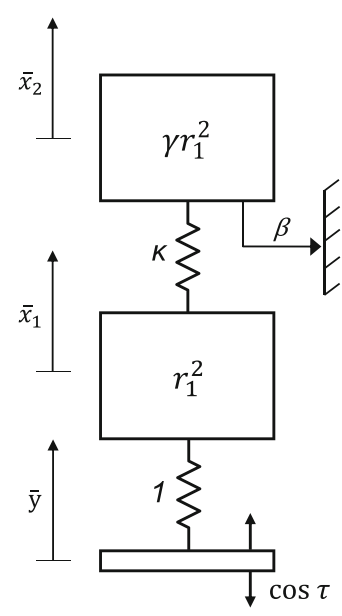

(b)
Fig. 2 Non-dimensional 2DOF system under harmonic base excitation with a Coulomb ground-fixed wall contact on a the lower mass or $\mathbf{b}$ the upper mass

also assumed that the magnitudes of static and kinetic friction forces are equal.

As several parameters appear in Eqs. (1) and (2), it is convenient to rewrite them in a non-dimensional form, using the smallest possible number of parameters required for describing the dynamic behaviour of the systems. A possible non-dimensional form of Eqs. (1) and (2) is:

$r_{1}^{2} \bar{x}_{1}^{\prime \prime}+(1+\kappa) \bar{x}_{1}-\kappa \bar{x}_{2}+\beta \operatorname{sgn}\left(\bar{x}_{1}^{\prime}\right)=\cos \tau$

$\gamma r_{1}^{2} \bar{x}_{2}^{\prime \prime}-\kappa \bar{x}_{1}+\kappa \bar{x}_{2}=0$

and:

$r_{1}^{2} \bar{x}_{1}^{\prime \prime}+(1+\kappa) \bar{x}_{1}-\kappa \bar{x}_{2}=\cos \tau$

$\gamma r_{1}^{2} \bar{x}_{2}^{\prime \prime}-\kappa \bar{x}_{1}+\kappa \bar{x}_{2}+\beta \operatorname{sgn}\left(\bar{x}_{2}^{\prime}\right)=0$

In the above equations, a non-dimensional time and a non-dimensional position for the $j$-th mass were introduced, respectively, as:

$$
\tau=\omega t \quad \bar{x}_{j}=\frac{x_{j}}{Y}
$$

and the symbol ' indicates the derivative with respect to $\tau$. The four non-dimensional groups chosen are:
- the frequency ratio:

$$
r_{1}=\omega \sqrt{\frac{m_{1}}{k_{1}}}
$$

- the friction ratio:

$$
\beta=\frac{F}{k_{1} Y}
$$

- the stiffness ratio:

$$
\kappa=\frac{k_{2}}{k_{1}}
$$

- the mass ratio:

$$
\gamma=\frac{m_{2}}{m_{1}}
$$

It is worth noting that Eqs. (4) and (5) can be interpreted as the governing equations of equivalent nondimensional systems where, as shown in Fig. 2a, b, the masses are $r_{1}^{2}$ and $\gamma r_{1}^{2}$ and the springs have a stiffness equal to 1 and $\kappa$, respectively. The friction ratio $\beta$ represents the amplitude of the friction force, while the base excitation is of unitary amplitude and unitary frequency.

\subsection{Sticking conditions}

The conditions for which a sticking phase will occur in the mass motion are discussed here. These conditions are required for the numerical integration of Eqs. (4) and (5) with the approach described in Sect. 4. Sticking will occur when, at a specific time, the relative velocity between the components in contact is zero and the amplitude of the sum of all the non-inertial forces acting on the mass in contact does not overcome the amplitude of the friction force. This translates into the conditions:

$\bar{x}_{1}^{\prime}=0$

$\left|\cos \tau-(1+\kappa) \bar{x}_{1}+\kappa \bar{x}_{2}\right|<\beta$

for the system in Fig. 2a and in:

$\bar{x}_{2}^{\prime}=0$

$\kappa\left|\bar{x}_{2}-\bar{x}_{1}\right|<\beta$ 
for the system in Fig. 2b.

\subsection{Boundaries of motion regimes for a SDOF system}

The boundary between continuous and stick-slip motion regions for MDOF systems will be determined as an extension of the expression found by Den Hartog for SDOF systems with Coulomb damping [2]. Within Den Hartog's approach:

- the Coulomb friction force is expressed as $-F \operatorname{sgn}(\dot{x})$, where $\dot{x}$ is the relative velocity between the mass and the wall. This force introduces a nonlinearity in the problem only if the velocity sign changes in a certain time interval;

- a steady-state response period included between two subsequent response maxima is considered. Assuming that the motion is continuous, the minimum displacement will occur in the middle of the interval, so the velocity sign will be constant and negative if only the first half cycle is taken into account;

- therefore, a linear problem is defined for this subinterval and an analytical solution for mass motion is found, allowing the determination of closed-form expressions of the amplitude and of the phase angle of the response;

- the conditions for which a stop occurs inside this time interval are used to evaluate a closed-form expression of the upper bound for non-sticking motion.

For each frequency ratio $r$, the smallest friction ratio for which a stop occurs inside the considered time interval is expressed as:

$\beta_{\lim }=\sqrt{\frac{V^{2}}{U^{2}+\left(S / r^{2}\right)^{2}}}$

The following quantities are introduced in the above equation:

- the response function:

$$
V=\frac{1}{1-r^{2}}
$$

is the frequency response of an undamped SDOF system;
- the damping function:

$$
U=\frac{\sin (\pi / r)}{r[1+\cos (\pi / r)]}
$$

describes the friction effect on the frequency response of the system;

- the function:

$$
S=\max _{0 \leq \tau \leq \pi} \frac{r\{\sin (\tau / r)+U r[\cos \tau-\cos (\tau / r)]\}}{\sin \tau}
$$

has been observed to be unitary for most values of $r$ [2] and, therefore, the assumption of $S=1$ will be considered in what follows. This assumption eliminates the time dependence of Den Hartog's boundary and reduces Eq. (13) to the solution presented by Hong and Liu in reference [5], which has been obtained with a different analytical approach.

It is worth noting that Den Hartog's boundary was obtained under the assumption of steady-state motion. In reference [4], Shaw demonstrated that SDOF systems with Coulomb friction are asymptotically stable in the absence of viscous damping, except that for $r=1 / n, n=1,2, \ldots$; particularly, an infinity of equally marginally stable solutions coexist if $r=1 /(2 n)$ [6]. Therefore, excluding these particular values, different motion regimes cannot coexist for given $r$ and $\beta$ depending on the initial conditions. Moreover, it must be observed that for $r=1$ the amplitude of the response will grow indefinitely if $\beta<\pi / 4$ [2] and, therefore, steady-state condition will not be reached.

Continuous motion will occur below the boundary described by Eq. (13) and is depicted by the blue area in Fig. 3, while stick-slip motion is expected above this line (the orange area in Fig. 3). Steady mass motion will not be possible when the amplitude of the exciting force is smaller than the amplitude of the static friction force; this happens when $\beta \geq 1$ (grey area). This basic notion will be used in more complex systems to obtain the boundary between motion and no motion regions. 


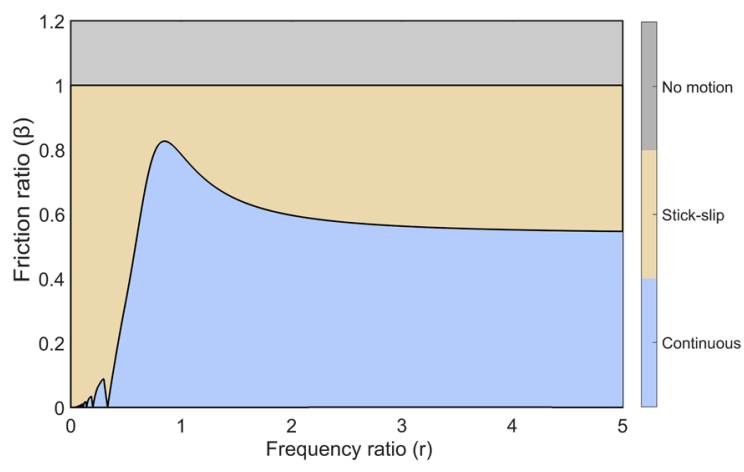

Fig. 3 Motion regimes of a SDOF system under harmonic excitation with a Coulomb fixed wall contact in the parameter space $r-\beta$

\subsection{Boundary between continuous and stick-slip regimes for $2 \mathrm{DOF}$ systems}

The analytical approach proposed for the evaluation of the boundary between continuous and stick-slip motion regimes in MDOF systems with a Coulomb friction contact is based on the following assumptions and observations.

- It is assumed that the steady-state response of the system is independent of the assigned initial conditions and converges asymptotically to a stable solution. As previously mentioned, this stability property is well known for SDOF systems but, to the best of the authors' knowledge, it has never been thoroughly investigated in the MDOF case. The convergence to a unique steady-state response has been verified in all the numerical investigations carried out in this paper.

- If the relative motion between the mass and the wall in contact is non-sticking, the governing equations of the system will be linear within a time interval equal to half period of motion. In fact, the Coulomb force will be constant in any interval where no change in the sign of their relative velocity occurs.

- The response functions of the system can be obtained by neglecting the friction force and using a standard modal analysis procedure, as described in Sect. 2.4.1.

- In addition, this conjecture is proposed: the boundary between continuous and stick-slip regimes can be expressed by using Eq. (13), in the assumption of $S=1$. In this equation, the response function $V$ is obtained as described above. The damping function
$U$ is formulated in a similar fashion as a superposition of the damping functions of each vibrating mode.

Numerical investigations are carried out for varying parameters, masses in contact and numbers of DOFs to validate the boundaries obtained under these assumptions.

\subsubsection{Response functions}

Although the response functions of a MDOF system can be determined by using standard modal analysis, the main steps of the procedure will be reported in this section to define the relevant variables. The approach is described in detail for the 2DOF case and can be easily extended to systems with a larger number of degrees of freedom, as described in Sect. 2.6.

The first step consists in evaluating the natural frequencies and the corresponding mode shapes of the undamped system, therefore disregarding the friction effect and the external excitation. Let us denote as $\Omega_{i}$ the natural frequencies of the linear system in the physical coordinates space. The natural frequencies of the non-dimensional system can be expressed as:

$\bar{\Omega}_{i}=\frac{\Omega_{i}}{\omega}$

Such frequencies can be obtained as solutions of the generalised eigenvalue problem written in the form:

$\left(\overline{\mathbf{K}}-\bar{\Omega}_{i}^{2} \overline{\mathbf{M}}\right) \boldsymbol{\psi}=\mathbf{0}$

where

$\overline{\mathbf{M}}=\left[\begin{array}{cc}r_{1}^{2} & 0 \\ 0 & \gamma r_{1}^{2}\end{array}\right]$

and:

$\overline{\mathbf{K}}=\left[\begin{array}{cc}1+\kappa & -\kappa \\ -\kappa & \kappa\end{array}\right]$

are, respectively, the mass and the stiffness matrices of the non-dimensional system and where the corresponding mode shapes are indicated with $\psi_{i}=$ $\left[\begin{array}{ll}\psi_{1, i} & \psi_{2, i}\end{array}\right]^{T}$. Thus, the natural frequencies can be viewed as eigenvalues and the mode shapes as eigenvectors. For a non-trivial solution of Eq. (18), it is 
required that:

$\operatorname{det}\left(\overline{\mathbf{K}}-\bar{\Omega}_{1,2}^{2} \overline{\mathbf{M}}\right)=0$

which leads to an algebraic equation. The resulting natural frequencies can be written as:

$\bar{\Omega}_{1,2}=\frac{1}{r_{1} \sqrt{2 \gamma}}\left[(\gamma+\kappa+\gamma \kappa) \mp \sqrt{(\gamma+\kappa+\gamma \kappa)^{2}-4 \gamma \kappa}\right]^{\frac{1}{2}}$

Having found the natural frequencies, the mode shapes $\psi_{i}$ must satisfy:

$$
\begin{aligned}
& \left(1+\kappa-\bar{\Omega}_{i}^{2} r_{1}^{2}\right) \psi_{1, i}-\kappa \psi_{2, i}=0 \\
& -\kappa \psi_{1, i}+\left(\kappa-\gamma \bar{\Omega}_{i}^{2} r_{1}^{2}\right) \psi_{2, i}=0
\end{aligned}
$$

The mode shapes are defined up to a constant, so only the ratio between their components can be obtained from the system in Eq. (23):

$\varphi_{i}=\frac{\psi_{2, i}}{\psi_{1, i}}=\frac{1}{1-\frac{\gamma}{\kappa} \bar{\Omega}_{i}^{2} r_{1}^{2}}$

In order to define uniquely the components of each mode a normalisation is usually operated according to different criteria (see reference [30]). In this paper, the modes will be normalised so that the modal masses:

$\hat{m}_{i}=\boldsymbol{\psi}_{i}^{T} \overline{\mathbf{M}} \boldsymbol{\psi}_{i}$

are equal to 1 . The normalised mode shape vectors obtained from such procedure are:

$\boldsymbol{\psi}_{i}=\frac{1}{r_{1} \sqrt{1+\gamma \varphi_{i}^{2}}}\left[\begin{array}{ll}1 & \varphi_{i}\end{array}\right]^{T}$

These eigenvectors are independent and therefore any undamped motion of the system can be written as their linear combination. Let us define the modal matrix $\Psi$ as the matrix whose columns are the mode shapes:

$\boldsymbol{\Psi}=\left[\begin{array}{ll}\boldsymbol{\psi}_{1} & \boldsymbol{\psi}_{2}\end{array}\right]=\frac{1}{r_{1}}\left[\begin{array}{ll}\frac{1}{\sqrt{1+\gamma \varphi_{1}^{2}}} \frac{1}{\sqrt{1+\gamma \varphi_{2}^{2}}} \\ \frac{\varphi_{1}}{\sqrt{1+\gamma \varphi_{1}^{2}}} \frac{\varphi_{2}}{\sqrt{1+\gamma \varphi_{2}^{2}}}\end{array}\right]$

The modal matrix is used to introduce the coordinate transformation:

$\overline{\mathbf{x}}=\boldsymbol{\Psi} \bar{\eta}$

where the components $\bar{\eta}_{i}$ of the vector $\bar{\eta}$ are defined as modal coordinates. The introduction of this system of coordinates allows the rewriting of Eqs. (4) and (5) as systems of uncoupled equations. In fact, neglecting friction force at this stage, Eq. (4), as well as Eq. (5), can be written in matricial form as:

$\overline{\mathbf{M}} \overline{\mathbf{x}}^{\prime \prime}+\overline{\mathbf{K}} \overline{\mathbf{x}}=\overline{\mathbf{p}}$

where $\overline{\mathbf{p}}=\left[\begin{array}{ll}\cos \tau & 0\end{array}\right]^{T}$. By introducing the transformation in Eq. (28), the governing equations assume the form:

$\boldsymbol{\Psi}^{T} \overline{\mathbf{M}} \boldsymbol{\Psi} \bar{\eta}^{\prime \prime}+\boldsymbol{\Psi}^{T} \overline{\mathbf{K}} \boldsymbol{\Psi} \bar{\eta}=\boldsymbol{\Psi}^{T} \overline{\mathbf{p}}$

or, in a more compact form:

$\hat{\mathbf{M}} \bar{\eta}^{\prime \prime}+\hat{\mathbf{K}} \bar{\eta}=\hat{\mathbf{p}}$

where

$\hat{\mathbf{M}}=\Psi^{T} \overline{\mathbf{M}} \boldsymbol{\Psi}=\mathbf{I}$

and:

$\hat{\mathbf{K}}=\Psi^{T} \overline{\mathbf{K}} \boldsymbol{\Psi}=\operatorname{diag}\left(\bar{\Omega}_{i}^{2}\right)$

are, respectively, the modal mass and the modal stiffness matrices, while: 
$\hat{\mathbf{p}}=\boldsymbol{\Psi}^{T} \overline{\mathbf{p}}=\frac{1}{r_{1}} \cos \tau\left[\frac{1}{\sqrt{1+\gamma \varphi_{1}^{2}}} \frac{1}{\sqrt{1+\gamma \varphi_{2}^{2}}}\right]^{T}$

is defined as the modal force vector. Therefore, the $i$-th equation of the system in Eq. (31) can be written as:

$\bar{\eta}_{i}^{\prime \prime}+\bar{\Omega}_{i}^{2} \bar{\eta}_{i}=\hat{p}_{i}$

Equation (35) represents the governing equation of a SDOF system characterised by the natural frequency $\bar{\Omega}_{i}$. Therefore, the amplitude $\bar{H}_{i}$ of its response to the exciting force $\hat{p}_{i}$ can be expressed as:

$\bar{H}_{i}=\frac{\hat{P}_{i}}{\bar{\Omega}_{i}^{2}-1}=\frac{1}{r_{1} \sqrt{1+\gamma \varphi_{i}^{2}}} \frac{1}{\bar{\Omega}_{i}^{2}-1}$

where

$\hat{P}_{i}=\frac{1}{r_{1} \sqrt{1+\gamma \varphi_{i}^{2}}}$

is the amplitude of the $i$-th modal force. From Eq. (28), it can be observed that:

$\bar{x}_{j}=\psi_{j, 1} \bar{\eta}_{1}+\psi_{j, 2} \bar{\eta}_{2}=\sum_{i=1}^{2} \psi_{j, i} \bar{\eta}_{i}$

and, therefore, it is possible to obtain the response function for the $j$-th degree of freedom of the undamped system as:

$V_{j}=\sum_{i=1}^{2} \psi_{j, i} \bar{H}_{i}$

By introducing the $i$-th modal frequency ratio as:

$R_{i}=\frac{\omega}{\Omega_{i}}=\frac{1}{\bar{\Omega}_{i}}$

it is possible to write $V_{j}$ as:

$V_{j}=\sum_{i=1}^{2} \psi_{j, i} \hat{P}_{i} \frac{R_{i}^{2}}{1-R_{i}^{2}}$
It is worth noting as the excitation vector $\overline{\mathbf{p}}$ can assume different forms if different loading configurations are considered, e.g. when the harmonic excitation is applied to the upper mass. This case is not accounted in this section but it will be dealt with in Sects. 3 and 4. Let us introduce the modal weight:

$\Gamma_{P, j i}=\psi_{j, i} \hat{P}_{i} R_{i}^{2}$

and denote the response functions of the $i$-th mode as:

$v_{i}=\frac{1}{1-R_{i}^{2}}$

It is then possible to rewrite the $j$-th response function as:

$V_{j}=\sum_{i=1}^{2} \Gamma_{P, j i} v_{i}$

and, by introducing the matrix of the modal weights $\boldsymbol{\Gamma}_{\mathrm{P}}$ and the vector $\mathbf{v}$ whose components are $v_{i}$, the response vector as:

$\mathbf{V}=\boldsymbol{\Gamma}_{\mathrm{P}} \mathbf{v}$

This notation can be particularly useful when dealing with systems with a larger number of DOFs.

The response functions of a 2DOF system under harmonic base excitation, observed on the lower and on the upper mass, are obtained by substituting Eqs. (26) and (37) into Eq. (41) and can be written, respectively, as:

$V_{1}=\sum_{i=1}^{2} \frac{1}{1+\gamma \varphi_{i}^{2}}\left(\frac{R_{i}}{r_{1}}\right)^{2} \frac{1}{1-R_{i}^{2}}$

and:

$V_{2}=\sum_{i=1}^{2} \frac{\varphi_{i}}{1+\gamma \varphi_{i}^{2}}\left(\frac{R_{i}}{r_{1}}\right)^{2} \frac{1}{1-R_{i}^{2}}$

\subsubsection{Damping functions and results}

In this study, it is proposed that modal superposition can be used to express the damping functions of a MDOF system. 
In a similar fashion as in Eq. (15), let us denote the damping function of the $i$-th mode as:

$u_{i}=\frac{\sin \left(\pi / R_{i}\right)}{R_{i}\left[1+\cos \left(\pi / R_{i}\right)\right]}$

Let us suppose that the damping function of a $2 \mathrm{DOF}$ system with a ground-fixed wall contact on the $j$-th mass can be written as:

$U_{j}=\sum_{i=1}^{2} \Gamma_{F, j i} u_{i}$

where $\Gamma_{F, j i}$ is the friction modal weight relative to the $i$-th mode, expressed as:

$\Gamma_{F, j i}=\boldsymbol{\psi}_{i}^{T} \mathbf{e}_{\mathrm{F}} \psi_{j, i} R_{i}^{2}$

and $\mathbf{e}_{\mathrm{F}}$ is a vector where only the $j$-th component is different from zero and it is equal to 1. Comparing Eqs. (42) and (50), it is possible to note as in the latter the excitation vector $\overline{\mathbf{P}}$ is replaced by $\mathbf{e}_{\mathrm{F}}$. From Eq. (50), it is easily obtained that:

$\Gamma_{F, j i}=\psi_{j, i}^{2} R_{i}^{2}$

By introducing the matrix of the friction modal weights, whose coefficients are $\Gamma_{F, j i}$, the damping vector $\mathbf{U}$ of a MDOF system can be written as:

$\mathbf{U}=\boldsymbol{\Gamma}_{\mathrm{F}} \mathbf{u}$

The components $U_{j}$ of such vector will indicate the damping function that must be considered if a fixedwall contact is imposed on the $j$-th mass of the system.

An expression is proposed for the boundary between continuous and stick-slip motion in the space $r_{1}-\beta$. Denoting with $\beta_{j}$ the friction ratio relative to a contact between the mass $m_{j}$ and the wall, the boundary can be written in a similar fashion to Eq. (13) as:

$\beta_{j, \lim }=\sqrt{\frac{V_{j}^{2}}{U_{j}^{2}+\left(1 / \bar{m}_{j}\right)^{2}}}$

The term $\bar{m}_{j}$ refers to the second-order coefficient in the non-dimensional governing equations in Eqs. (4) and (5), i.e. $\bar{m}_{1}=r_{1}^{2}$ and $\bar{m}_{2}=\gamma r_{1}^{2}$. The damping function $U_{j}$ can be rewritten, by substituting Eqs. (48) and (51) into Eq. (49), as:

$U_{j}=\sum_{i=1}^{2} \psi_{j, i}^{2} R_{i}^{2} \frac{\sin \left(\pi / R_{i}\right)}{R_{i}\left[1+\cos \left(\pi / R_{i}\right)\right]}$

With respect to the system illustrated in Figs. 1a and $2 \mathrm{a}$, where the contact occurs between the lower mass $(j=1)$ and a fixed wall, the damping function will be therefore expressed as:

$U_{1}=\sum_{i=1}^{2} \frac{1}{1+\gamma \varphi_{i}^{2}}\left(\frac{R_{i}}{r_{1}}\right)^{2} \frac{\sin \left(\pi / R_{i}\right)}{R_{i}\left[1+\cos \left(\pi / R_{i}\right)\right]}$

and, consequently, the boundary will be expressed as:

$\beta_{1, \lim }=\sqrt{\frac{V_{1}^{2}}{U_{1}^{2}+\left(\frac{1}{r_{1}^{2}}\right)^{2}}}$

The boundary obtained from Eq. (56) is represented in Fig. 4 for different values of the mass and stiffness ratios. In the figure, it is shown as this analytical curve has an excellent agreement with the results obtained via numerical integration, using the approach described in Sect. 4 for $0 \leq r_{1} \leq 2.5$ and $0 \leq \beta \leq 1$. Stickslip motion occurs also for low friction ratios when the frequency ratio is small; furthermore, in the same frequency range, the boundary shows an irregular pattern, partially recalling the one observed in SDOF systems (Fig. 3). Nevertheless, a main difference is that a peak can always be observed in this range, specifically in correspondence of the lowest natural frequency of the system. Moving towards higher frequency ratios, it is possible to observe a very thin grey region (more clearly in Fig. 4a,d). This corresponds to an antiresonance of the system, which can be observed in the lower mass of a 2DOF system, independently of damping, at:

$r_{1}=\sqrt{\frac{\kappa}{\gamma}}$

At this frequency, in the presence of Coulomb damping, the friction prevents the system from exhibiting any vibration in steady-state conditions; therefore, no motion has been observed numerically. The right side of the boundary reproduces the same pattern observed 


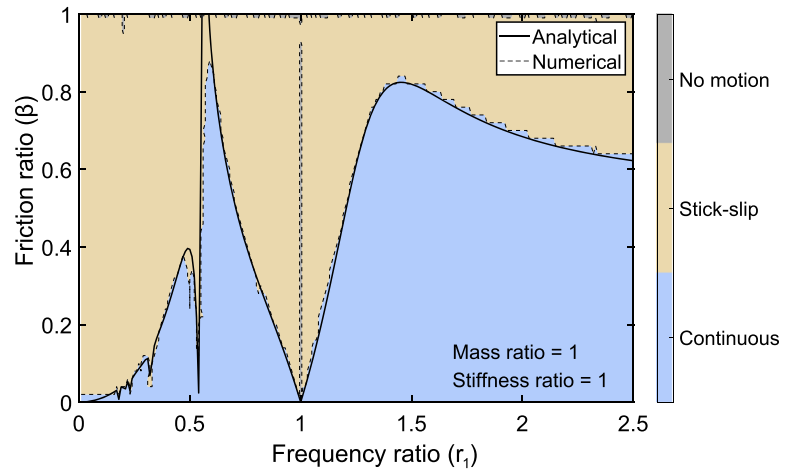

(a) $\gamma=1, \kappa=1$

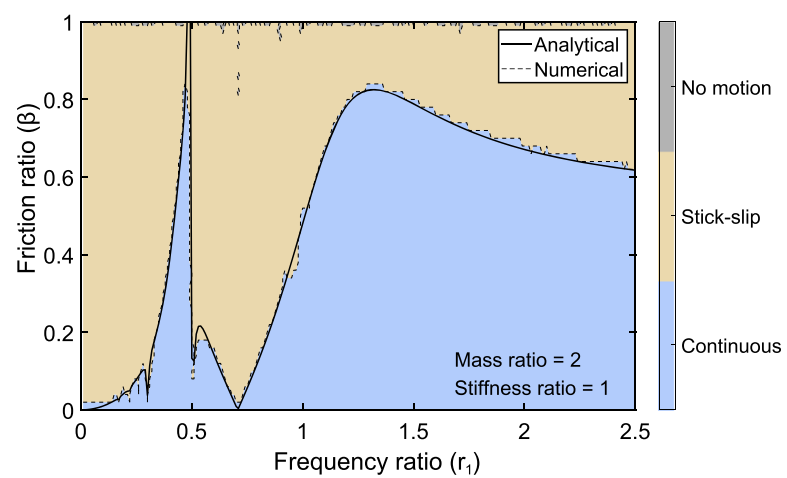

(c) $\gamma=2, \kappa=1$

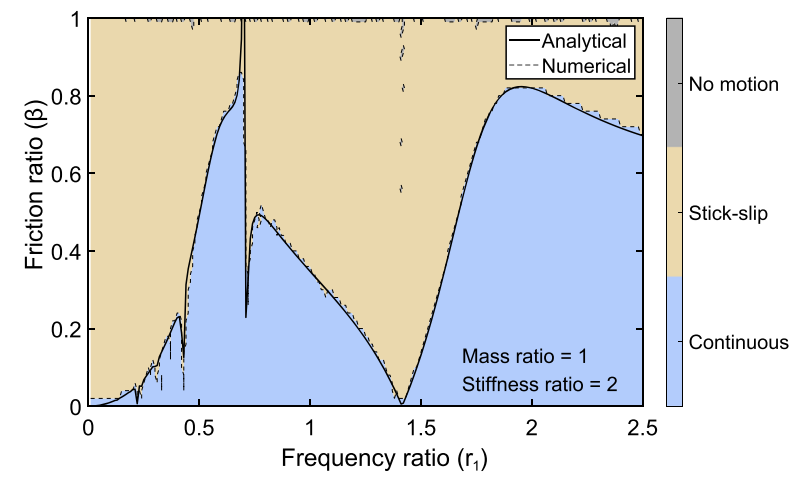

(b) $\gamma=1, \kappa=2$

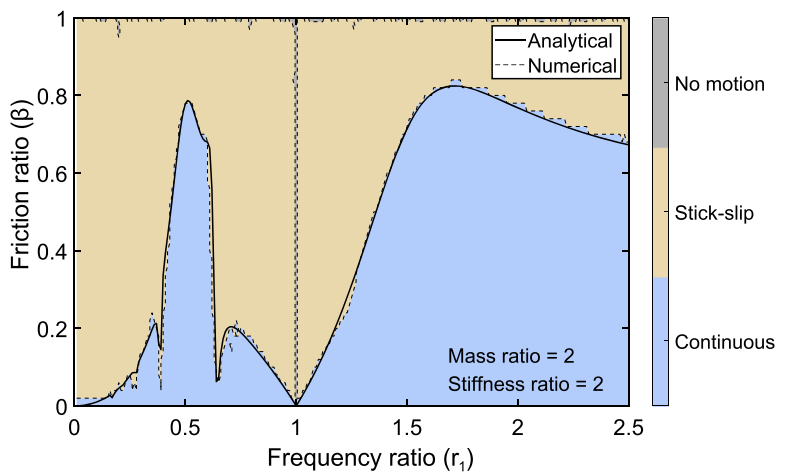

(d) $\gamma=2, \kappa=2$

Fig. 4 Motion regimes of a 2DOF system under harmonic excitation with a Coulomb ground-fixed wall contact on the lower mass in the parameter space $r_{1}-\beta$. Coloured regions refer to the numerical results. Each figure corresponds to a different mass ratio $(\gamma)$ and stiffness ratio $(\kappa)$

in SDOF systems, with a finite peak with $\beta \cong 0.8$, reached slightly before the second resonant frequency ratio of the system, and then decreasing towards an asymptotic value [10].

The same approach can also be applied to a 2DOF system where the ground-fixed wall contact involves the upper mass (Figs. 1b, 2b). In this case, the damping function and the boundary condition can be written, respectively, as:

$$
U_{2}=\sum_{i=1}^{2} \frac{\varphi_{i}^{2}}{1+\gamma \varphi_{i}^{2}}\left(\frac{R_{i}}{r_{1}}\right)^{2} \frac{\sin \left(\pi / R_{i}\right)}{R_{i}\left[1+\cos \left(\pi / R_{i}\right)\right]}
$$

and:

$$
\beta_{2, \lim }=\sqrt{\frac{V_{2}^{2}}{U_{2}^{2}+\left(\frac{1}{\gamma r_{1}^{2}}\right)^{2}}}
$$




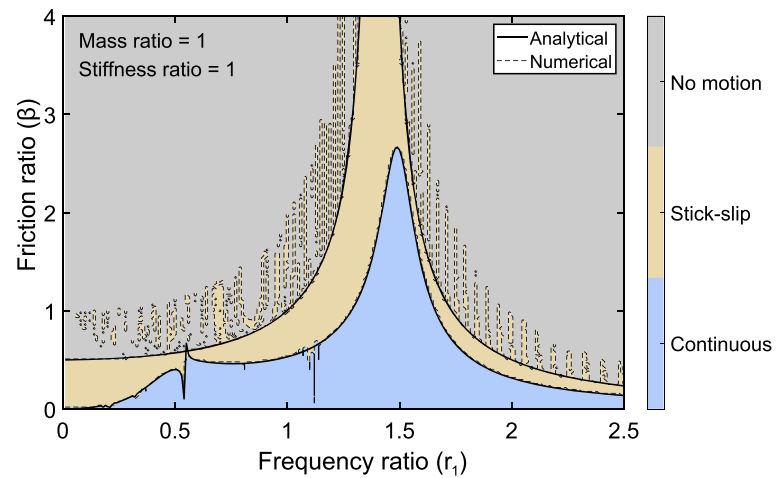

(a) $\gamma=1, \kappa=1$

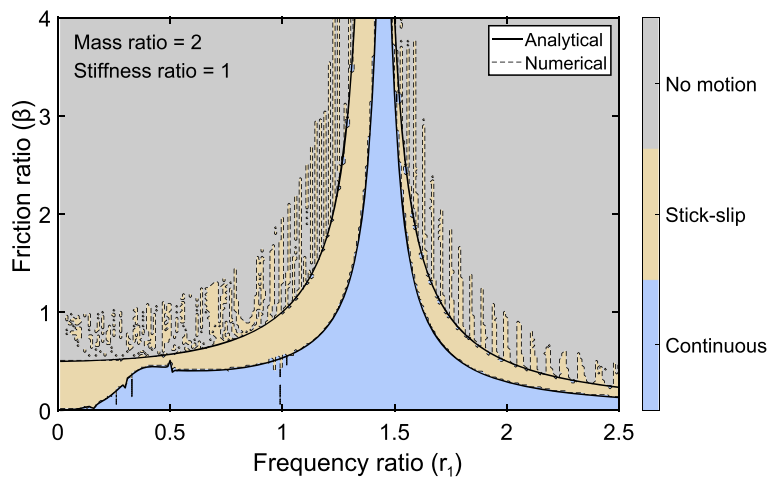

(c) $\gamma=2, \kappa=1$

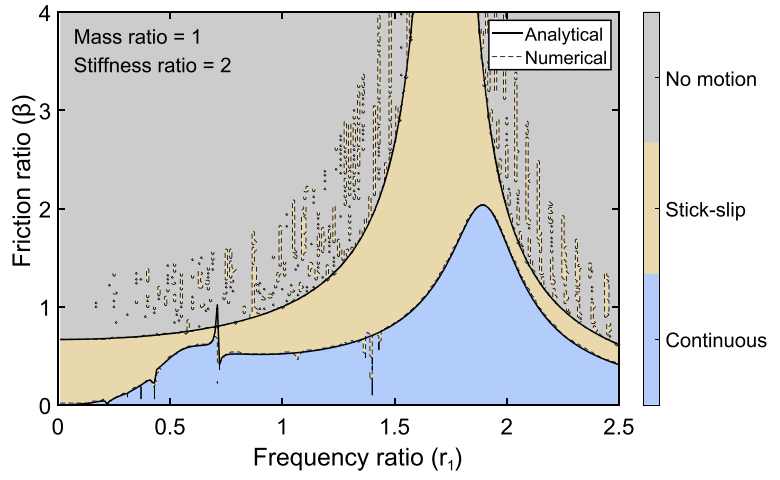

(b) $\gamma=1, \kappa=2$

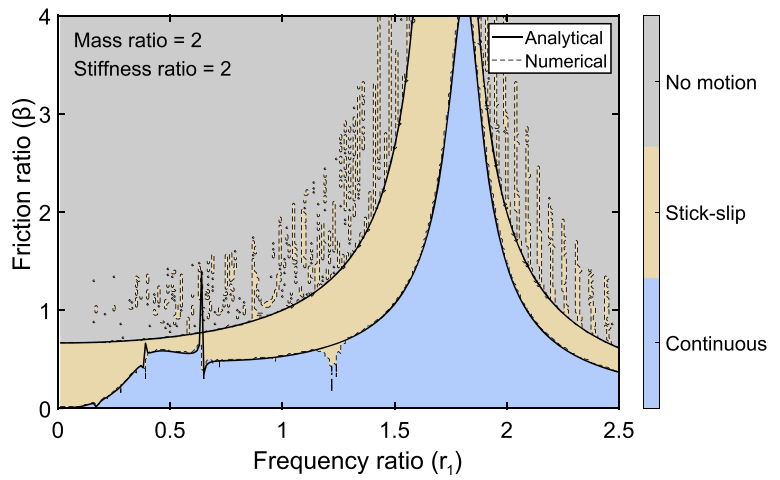

(d) $\gamma=2, \kappa=2$

Fig. 5 Motion regimes of a 2DOF system under harmonic excitation with a Coulomb ground-fixed wall contact on the upper mass in the parameter space $r_{1}-\beta$. Coloured regions refer to the numerical results. Each figure corresponds to a different mass ratio $(\gamma)$ and stiffness ratio $(\kappa)$

This analytical function is shown in Fig. 5, where it is compared with the numerical boundary between continuous and stick-slip motion, showing also in this case an excellent agreement. Particularly, the boundary appears to increase from zero to a finite peak, although not regularly at low frequencies. This peak is located between the peak of the boundary between motion and no motion regions (described in Sect. 2.5) and the second natural frequency of the system. It is also possible to observe as the frequency ratio of the peak appears to be only weakly influenced by the mass ratio. Finally, increasing $r_{1}$ above the peak frequency ratio, the boundary converges to zero. Some irregularities in the agreement between analytical and numerical boundaries can be observed locally (for instance at $r \cong 1.2$ in Fig. $5 \mathrm{~d}$ ); this is due to the approximation introduced by assuming $S=1$, as specified in Sect. 2.3.

\subsection{Condition for the presence of a no motion region in 2DOF systems}

In Fig. 5, numerical results revealed a large no motion region, shown in grey.

As stated in Sect. 2.3 for SDOF systems, steady-state response can be observed in Coulomb damped systems only when the amplitude of the exciting force is larger than the amplitude of the friction force. Specifically, in MDOF systems with a single source of Coulomb damping, this condition must be verified on the mass directly involved in the contact by comparing the amplitudes of the friction force exerted by the fixed wall and of the overall dynamic load acting on such mass when its displacement and velocity are equal to zero. Therefore, the conditions for steady motion between such mass $m_{i}$ and the wall in contact can be found assuming that the mass is perfectly fixed to the wall. 
For instance, when a friction contact between the lower mass and the wall is considered (Fig. 2a), the only force acting on $m_{1}$, in addition to the friction force, is the base excitation transmitted by the lower spring. As the amplitude of the non-dimensional base motion and the stiffness of this spring are both unitary, the motion condition will be given, trivially, by $\beta<1$, as observed for SDOF systems.

Instead, when the contact occurs on the second mass, the only exciting force to be considered is the spring force due to the displacement of the lower mass and transmitted by the upper spring. Thus, the condition for the presence of a steady motion is expressed by:

$\left|\kappa \bar{X}_{1}\right|>\beta$

The amplitude $\bar{X}_{1}$ of the lower mass motion can be evaluated by fixing the upper mass in the non-dimensional system (Fig. 2b). In this case, the system reduces to a SDOF, where the lower mass is attached to the ground on either side, by springs of stiffness, respectively, 1 and $\kappa$. Therefore, its governing equation will be:

$r_{1}^{2} \bar{x}_{1}^{\prime \prime}+(1+\kappa) \bar{x}_{1}=\cos \tau$

By imposing $\bar{x}_{1}=\bar{X}_{1} \cos \tau$, it is possible to write the response amplitude as:

$\bar{X}_{1}=\frac{1}{1+\kappa-r_{1}^{2}}$

Substituting Eq. (62) into Eq. (60), it is possible to rewrite the motion condition as:

$\beta<\frac{\kappa}{\left|1+\kappa-r_{1}^{2}\right|}$

This analytical boundary is plotted in Fig. 5 and shows a very good agreement with the boundary obtained from the numerical integration. A first observation is that this boundary is completely independent of the mass ratio; this justifies also the already mentioned weak dependence on $\gamma$ shown by the peak of the boundary between continuous and stick-slip regimes. It is possible to observe how the motion is allowed at $r \cong 0$ for force ratios smaller than $\kappa /(1+\kappa)$. The boundary increases monotonically until reaching an infinite peak for $r_{1}=\sqrt{1+\kappa}$, which is, therefore, the only frequency ratio for which motion is always allowed.
Table 1 Motion regimes of Coulomb damped 2DOF systems with a ground-fixed wall configuration for different friction ratio ranges

\begin{tabular}{lll}
\hline Contact & Friction ratio range & Regime \\
\hline$m_{1}$ & $0 \leq \beta<\sqrt{\frac{V_{1}^{2}}{U_{1}^{2}+\frac{1}{r_{1}^{4}}}}$ & Continuous \\
& $\sqrt{\frac{V_{1}^{2}}{U_{1}^{2}+\frac{1}{r_{1}^{4}}}} \leq \beta<1$ & Stick-slip \\
$\beta \geq 1$ & No motion \\
$m_{2}$ & $0 \leq \beta<\sqrt{\frac{V_{2}^{2}}{U_{2}^{2}+\frac{1}{\gamma^{2} r_{1}^{4}}}}$ & Continuous \\
& $\sqrt{\frac{V_{2}^{2}}{U_{2}^{2}+\frac{1}{\gamma^{2} r_{1}^{4}}}} \leq \beta<\frac{\kappa}{\left|1+\kappa-r_{1}^{2}\right|}$ & Stick-slip \\
$\beta \geq \frac{\kappa}{\left|1+\kappa-r_{1}^{2}\right|}$ & No motion \\
\hline
\end{tabular}

Finally, the boundary converges to zero at high frequencies. It is worth observing that the several spikes shown by the numerical results in Fig. 5 above the boundary are due to residual transient motion not completely decayed at the end of the time interval considered in the numerical simulation, as underlined in Sect. 5.

The motion regimes scenario defined by the analytical boundaries found in this section for 2DOF systems with a ground-fixed wall Coulomb contact is summarised in Table 1.

2.6 Boundaries for systems with more than two DOFs

The procedures described in Sects. 2.4 and 2.5 for the analytical determination of the boundaries between motion regimes for $2 \mathrm{DOF}$ systems with a fixed Coulomb contact can be extended to systems with a larger number of DOFs, maintaining the limitation that Coulomb damping must be generated by a single contact between one of the masses and the ground-fixed wall.

\subsubsection{Governing equations}

First of all, it is important to define the governing equations of a generic MDOF system consistently with the formulation used for 2DOF systems so far. Consider a NDOF system, made of $N$ masses $m_{i}$ connected in series by $N$ springs of stiffness $k_{i}$, which is subjected to a monoharmonic excitation with driving frequency $\omega$, due to either a base motion or a direct mass excitation 
(Fig. 6a). If a friction contact is imposed between the $j$-th mass and a fixed wall, it will be possible to write the $j$-th governing equation of the system as:

$$
\begin{aligned}
& m_{j} \ddot{x}_{j}-k_{j} x_{j-1} \\
& +\left(k_{j}+k_{j+1}\right) x_{j}-k_{j+1} x_{j+1}+F \operatorname{sgn}\left(\dot{x}_{j}\right)=p_{j}
\end{aligned}
$$

where $x_{j-1}=0$ if $j=1$ and $x_{j+1}=k_{j+1}=0$ if $j=N$. If present, the load $k_{1} y$ due to the base motion must be included in the equation if $j=1$. Equation (64) can also be written in non-dimensional form as:

$$
\begin{aligned}
& \gamma_{j} r_{1}^{2} \bar{x}_{j}^{\prime \prime}-\kappa_{j} \bar{x}_{j-1} \\
& \quad+\left(\kappa_{j}+\kappa_{j+1}\right) \bar{x}_{j}-\kappa_{j+1} \bar{x}_{j+1}+\beta \operatorname{sgn}\left(\bar{x}_{j}^{\prime}\right)=\bar{p}_{j}
\end{aligned}
$$

where the $j$-th mass ratio and the $j$-th stiffness ratio are defined, respectively, as:

$\gamma_{j}=\frac{m_{j}}{m_{1}}$

and:

$\kappa_{j}=\frac{k_{j}}{k_{1}}$

The non-dimensional system described by Eq. (65) is shown in Fig. 6b. It can be observed that the system is completely described by $2 N$ parameters: the frequency ratio $r_{1}$, the friction ratio $\beta$, the mass ratios $\gamma_{2}, \ldots, \gamma_{N}$ and the stiffness ratios $\kappa_{2}, \ldots, \kappa_{N}$. Trivially, $\gamma_{1}=1$ and $\kappa_{1}=1$ by definition.

\subsubsection{Boundary between continuous and stick-slip regimes}

Regarding the boundary between continuous and stickslip regimes, it is intuitive that the modal superposition can be applied to any number of DOFs. According to Eq. (65), the mass and the stiffness matrices will be, respectively:

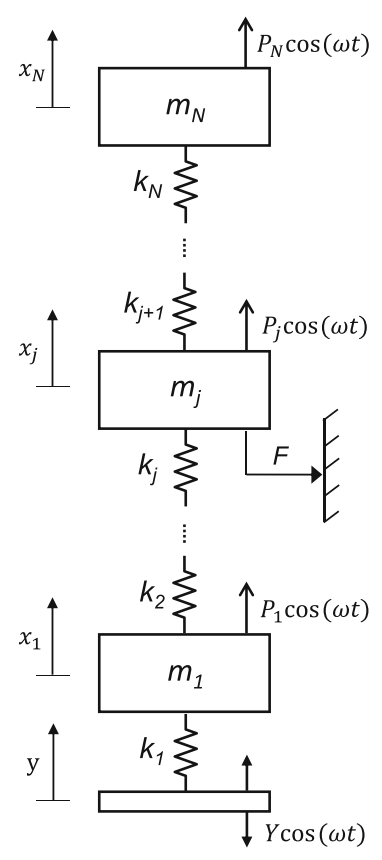

(a)
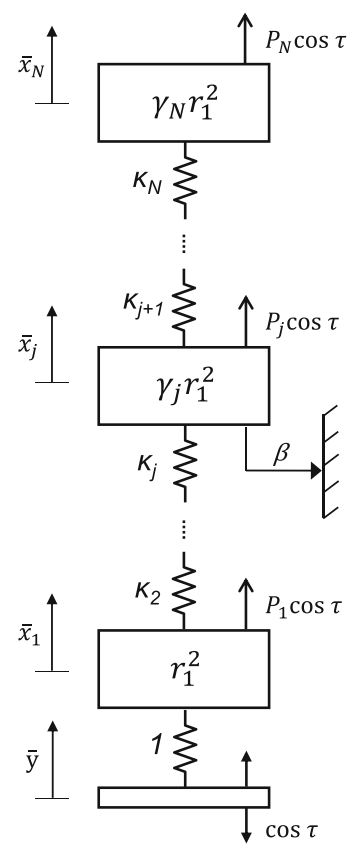

(b)
Fig. 6 NDOF system under harmonic excitation with a Coulomb ground-fixed wall contact on the $j$-th mass (a) and the correspondent non-dimensional system (b)

$\overline{\mathbf{M}}=\left[\begin{array}{cccc}r_{1}^{2} & 0 & \ldots & 0 \\ 0 & \gamma_{2} r_{1}^{2} & \ldots & 0 \\ \vdots & \vdots & \vdots & \vdots \\ 0 & 0 & \ldots & \gamma_{N} r_{1}^{2}\end{array}\right]$

and:

$\overline{\mathbf{K}}=\left[\begin{array}{ccccc}1+\kappa_{2} & -\kappa_{2} & 0 & \ldots & 0 \\ -\kappa_{2} & \kappa_{2}+\kappa_{3} & -\kappa_{3} & \ldots & 0 \\ \vdots & \vdots & \vdots & \vdots & \vdots \\ 0 & 0 & \ldots & -\kappa_{N} & \kappa_{N}\end{array}\right]$

By substituting these matrices into Eq. (22), it is possible to derive the $N$ natural frequencies of the undamped system and, from Eq. (21), its $N$ mode shapes. Once these quantities are determined, it is possible to follow the remaining part of the procedure described in Sect. 2.4, determining the response function $V_{j}$ and the damping function $U_{j}$ for the $j$-th mass. The boundary curve is finally obtained by substituting these values, as well as posing $\bar{m}_{j}=\gamma_{j} r_{1}^{2}(j=1, \ldots, N)$, into Eq. (53). 


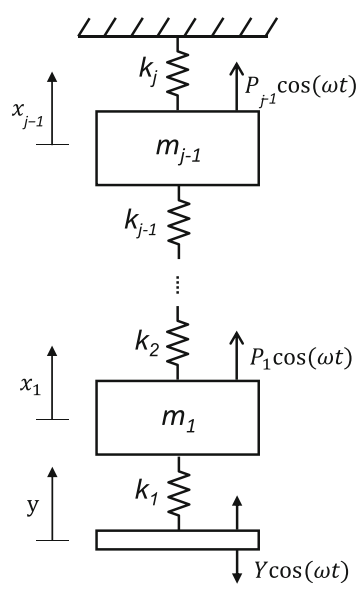

(a)

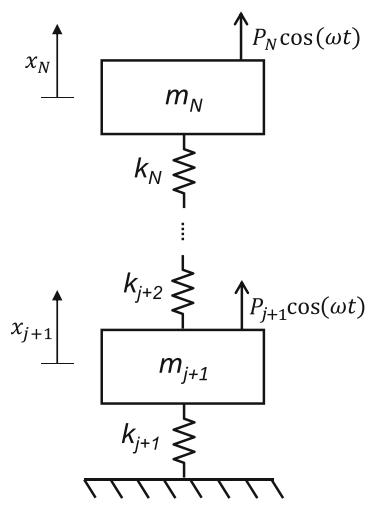

(b)
Fig. 7 Lower (a) and upper (b) subsystems for the evaluation of the motion conditions for a NDOF system under harmonic excitation with a Coulomb ground-fixed wall contact on the $j$-th mass

\subsubsection{Boundary between motion and no-motion regimes}

The determination of the boundary between motion and no motion regimes can be lead similarly to Sect. 2.5. The first step consists in determining which dynamic forces act on the mass in contact $m_{j}$ when it is fixed at $\bar{x}_{j}=0$. The sum of these forces, which will be compared with the friction force, can include, in general, dynamic loads applied directly on the mass and the spring forces due to the dynamic responses of the masses $m_{j-1}$ and $m_{j+1}$. Particularly:

- a spring force of module $\kappa_{j} \bar{X}_{j-1}$ will be considered if any source of excitation is found in the lower part of the system (Fig. 7a);

- a spring force of module $\kappa_{j+1} \bar{X}_{j+1}$ will be considered if any source of excitation is found in the upper part of the system (Fig. 7b);

The second step consists in the evaluation of the unknown response amplitudes $\bar{X}_{j-1}$ and/or $\bar{X}_{j+1}$, which can be obtained by referring to the following undamped subsystems:

- the response amplitude $\bar{X}_{j-1}$ can be evaluated from the lower subsystem, which is composed of the $j-1$ masses located below the mass in contact $m_{j}$, while such a mass is replaced by a fixed wall, as shown in Fig. 7a;

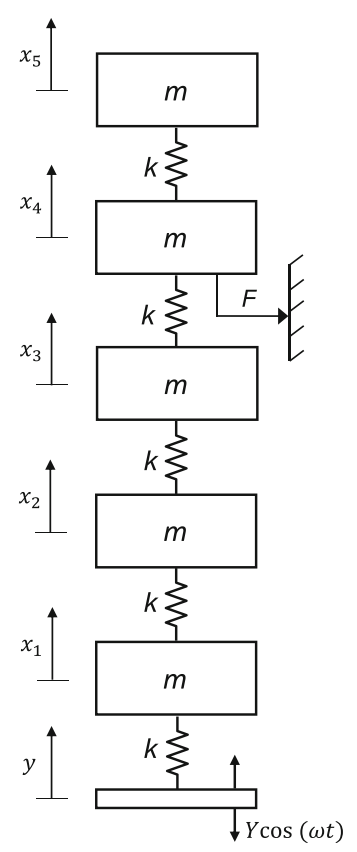

(a)

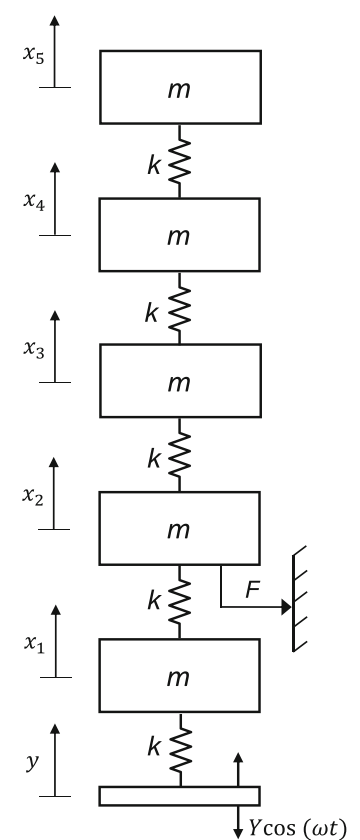

(b)
Fig. 8 5DOF system under harmonic excitation with a single Coulomb ground-fixed wall contact on the fourth mass (a) and on the second mass (b)

- the response amplitude $\bar{X}_{j+1}$ can be evaluated from the upper subsystem shown in Fig. 7b, where the $N-j$ upper masses are instead considered.

These subsystems will be, in general, two undamped MDOF systems and their dynamic response can be determined analytically using standard approaches (see, e.g. [29,30]).

These responses can be substituted into the motion conditions obtained at the end of the first step, therefore yielding the final formulation of the motion boundary. It can be observed that the response amplitudes evaluated from each of the subsystems will display infinite peaks in correspondence of their natural frequencies and this will affect the shape of the boundary: if the excitation is below $m_{j}$ (such as a base motion), the boundary between motion and no motion regimes will exhibit $j-1$ infinite peaks. Similarly, $N-j$ infinite peaks will be visualised if any dynamic force is acting on the upper part of the system; finally, $N-1$ peaks will be found if both loading conditions occur simultaneously. These infinite peaks imply that, at specific frequencies, 
steady-state motion will be observed in the contact for any values of friction ratio.

\subsubsection{Example: 5DOF systems with a ground-fixed Coulomb contact}

The procedure introduced for the analytical determination of the boundaries of motion regimes is applied to a 5DOF system under harmonic base motion with a Coulomb contact as an example of NDOF system with $N>2$.

Without loss of generality, let us consider a 5DOF system where all the masses are equal to $m$ and all the springs have stiffness $k$, i.e. where all the stiffness and mass ratios are unitary. A ground-fixed contact, characterised by a friction force of amplitude $F$, is applied to the mass $m_{4}$ and the system is subjected to a base motion $y=Y \cos (\omega t)$, as shown in Fig. 8a.

The response and the damping functions $V_{4}$ and $U_{4}$ have been evaluated by applying the modal superposition procedure introduced in this section and the boundary between continuous and stick-slip motion has been obtained by substituting their values into Eq. (53) for $j=4$. Regarding the boundary between motion and no motion regimes, it can be observed that the only dynamic force acting on $m_{4}$, when fixed at $x_{4}=0$, is a spring force of amplitude $k X_{3}$. Therefore, the boundary is obtained when $F=k X_{3}$ or, non-dimensionally, when $\beta=\bar{X}_{3}$. The value of $\bar{X}_{3}$ can be determined by using standard modal analysis on the undamped subsystem shown in Fig. 9a.

The so-determined boundaries are shown in Fig. $10 \mathrm{a}$, where a comparison with the numerical boundaries obtained with the approach introduced in Sect. 5 is also achieved, exhibiting a very good overall agreement. As already mentioned for other results, the small spikes present in the grey area are due to a not completely decayed transient motion in the numerical solutions, while the presence of some local disagreement in the boundary between continuous and stick-slip regimes at $r_{1} \cong 1.5$ is instead related to the approximation of $S=1$ (see Sect. 2.3).

As expected, the boundary between motion and no motion regions exhibits three infinite peaks for $r_{1}=$ $0.7654, r_{1}=1.4142$ and $r_{1}=1.8478$. Such peaks correspond to the resonances of the 3DOF undamped subsystem in Fig. 9a.

If the friction contact is applied on the second mass, as shown in Fig. 8b, the corresponding lower subsystem

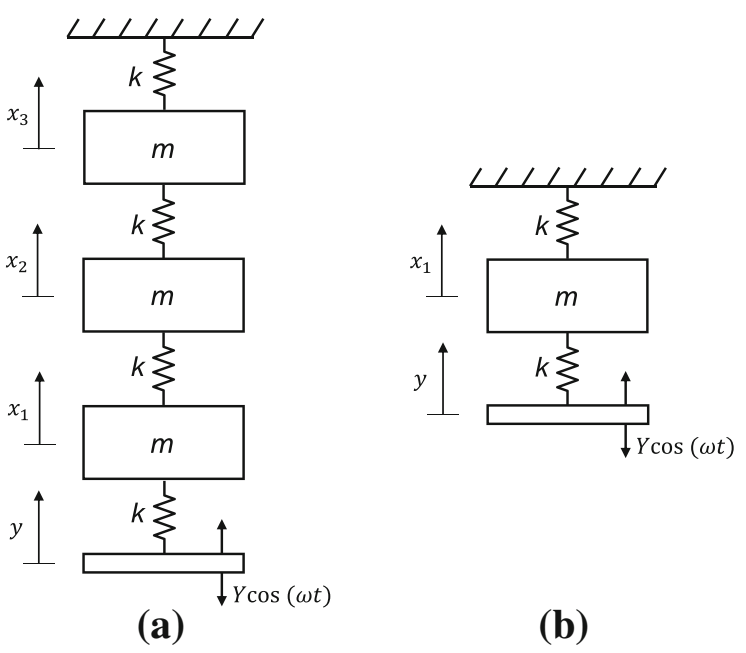

Fig. 9 Lower subsystems corresponding to the no-motion configurations of the main systems in Fig. 8a (a) and in Fig. 8b (b)

has only one DOF (Fig. 9b) and a single infinite peak is found (at $r_{1}=\sqrt{2}$ ) in the motion boundary plotted in Fig. 10b. In the figure, it is possible to observe a good agreement between analytical and numerical results; all the observations regarding the discrepancies between these results made for the previous system also apply to this configuration.

Finally, it can be observed in both cases that the shape of the upper bound for non-sticking motion is strongly affected by the presence of resonances in the motion/no motion boundary and usually exhibits the same number of major peaks; however, in all the cases investigated for the fixed-wall configuration, their value was always finite.

\section{Base-fixed wall contacts}

In this section, the analytical formulation of the boundaries achieved for MDOF systems with a ground-fixed wall contact is extended to systems where a Coulomb contact occurs between a mass and a wall moving jointly with the base. As proposed in reference [10] for SDOF systems, Den Hartog results [2] can be extended to systems excited by joined base-wall motion if an appropriate reference system is chosen. The analytical bounds of the motion regimes for 2DOF systems with base-fixed wall contacts are derived in what follows. 


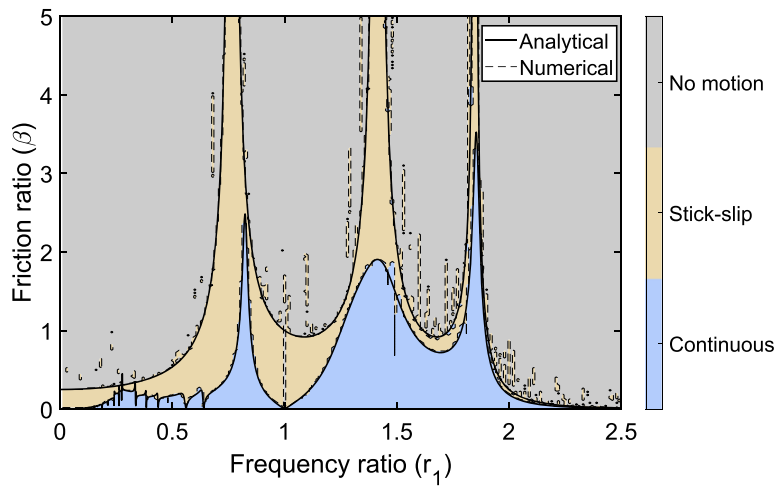

(a)

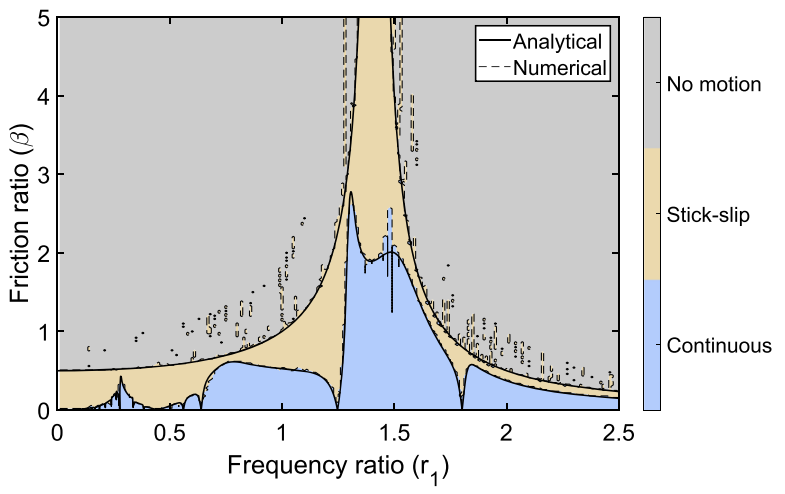

(b)

Fig. 10 Motion regimes of a 5DOF system under harmonic excitation with a single Coulomb ground-fixed wall contact on the fourth mass (a) and on the second mass (b) in the parameter space $r_{1}-\beta$, for mass ratios $\gamma_{2}=\gamma_{3}=\gamma_{4}=\gamma_{5}=1$ and stiffness ratios $\kappa_{2}=\kappa_{3}=\kappa_{4}=\kappa_{5}=1$. Coloured regions refer to the numerical results

\subsection{Governing equations and sticking conditions}

Let us consider a 2DOF system consisting of two masses $m_{1}$ and $m_{2}$ and two springs of stiffness $k_{1}$ and $k_{2}$. The system is assumed to be excited by a harmonic base motion $y=Y \cos (\omega t)$ and a friction contact is achieved between a moving wall jointed to the base and either $m_{1}$ (Fig. 11a) or $m_{2}$ (Fig. 11b). Such a system is governed by the equations:

$$
\begin{aligned}
& m_{1} \ddot{x}_{1}+\left(k_{1}+k_{2}\right) x_{1} \\
& \quad-k_{2} x_{2}+F \operatorname{sgn}\left(\dot{x}_{1}-\dot{y}\right)=k_{1} y \\
& m_{2} \ddot{x}_{2}-k_{2} x_{1}+k_{2} x_{2}=0
\end{aligned}
$$

in the first configuration and by the equations:

$m_{1} \ddot{x}_{1}+\left(k_{1}+k_{2}\right) x_{1}-k_{2} x_{2}=k_{1} y$

$m_{2} \ddot{x}_{2}-k_{2} x_{1}+k_{2} x_{2}+F \operatorname{sgn}\left(\dot{x}_{2}-\dot{y}\right)=0$

in the latter. In order to apply to this system the procedures described in Sect. 2, it is convenient to rewrite Eqs. (70) and (71) in the same form as Eqs. (1) and (2); this can be achieved by applying an appropriate variable transformation.

Let us define the relative motions between either mass $m_{1}$ or $m_{2}$, respectively, as:

$z_{1}=x_{1}-y$

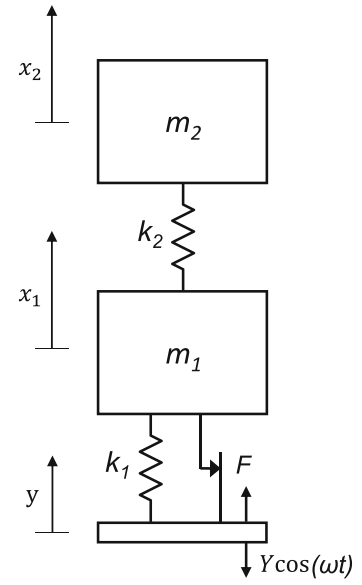

(a)

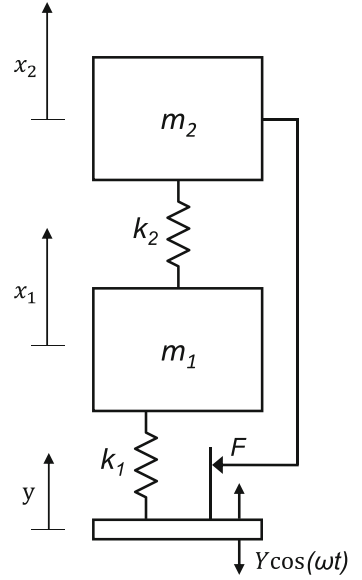

(b)
Fig. 11 2DOF system under harmonic base excitation with a Coulomb contact between a base-fixed wall and a the lower mass or $\mathbf{b}$ the upper mass

and:

$z_{2}=x_{2}-y$

. Substituting Eqs. (72) and (73) into Eqs. (70) and (71), and after some algebraic manipulations, it is possible to write:

$$
\begin{aligned}
& m_{1} \ddot{z}_{1}+\left(k_{1}+k_{2}\right) z_{1}-k_{2} z_{2}+F \operatorname{sgn}\left(\dot{z}_{1}\right)=k_{1} r_{1}^{2} y \\
& m_{2} \ddot{z}_{2}-k_{2} z_{1}+k_{2} z_{2}=k_{1} \gamma r_{1}^{2} y
\end{aligned}
$$




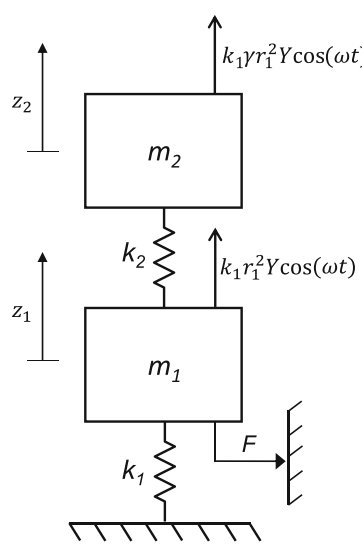

(a)

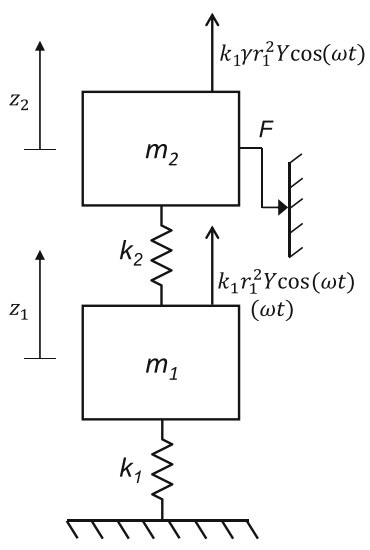

(b)
Fig. 12 Equivalent system with a ground-fixed wall contact for a $2 \mathrm{DOF}$ system with a base-fixed wall contact involving a the lower mass or $\mathbf{b}$ the upper mass

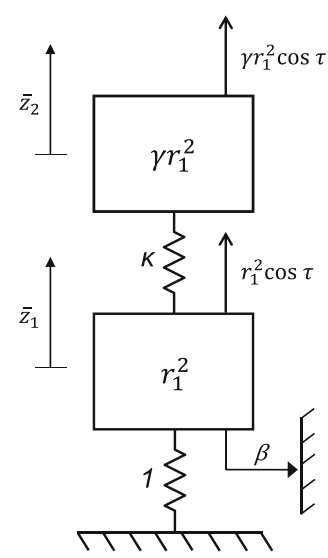

(a)

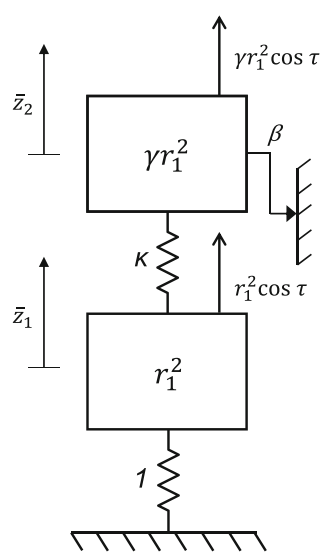

(b)
Fig. 13 Non-dimensional equivalent system with a ground-fixed wall contact for a $2 \mathrm{DOF}$ system with a base-fixed wall contact involving $\mathbf{a}$ the lower mass or $\mathbf{b}$ the upper mass

when the contact occurs between the base-jointed wall and the lower mass and:

$$
\begin{aligned}
& m_{1} \ddot{z}_{1}+\left(k_{1}+k_{2}\right) z_{1}-k_{2} z_{2}=k_{1} r_{1}^{2} y \\
& m_{2} \ddot{z}_{2}-k_{2} z_{1}+k_{2} z_{2}+F \operatorname{sgn}\left(\dot{z}_{2}\right)=k_{1} \gamma r_{1}^{2} y
\end{aligned}
$$

when the upper mass is in contact.

Equations (74) and (75) are the governing equations of the systems shown in Fig. 12a, b, which will be defined equivalent systems of the systems introduced in Fig. 11a, b. These 2DOF equivalent systems present a ground-fixed wall contact and, therefore, the modal superposition procedure can be applied as described in Sect. 2.4. As it can be observed from Fig. 12a, b, both masses are excited by equivalent harmonic forces whose amplitudes are proportional to $r_{1}^{2}$; therefore, the dynamic load will increase significantly at high frequency ratios, unlike the friction force, allowing the presence of continuous motion also when high friction ratios are considered. This result is in perfect agreement with what was observed in [10] for Coulombdamped SDOF systems under harmonic joined basewall motion.

In order to apply the modal superposition procedure, it is convenient to rewrite Eqs. (74) and (75) in a nondimensional form. Introducing the dimensionless state variables:

$\bar{z}_{1}=\frac{z_{1}}{Y} \quad \bar{z}_{2}=\frac{z_{2}}{Y}$

and considering all the non-dimensional groups introduced in Sect. 2.1, it is possible to write, with the respect to the two different contact locations considered in this section:

$r_{1}^{2} \bar{z}_{1}^{\prime \prime}+(1+\kappa) \bar{z}_{1}-\kappa \bar{z}_{2}+\beta \operatorname{sgn}\left(\bar{z}_{1}^{\prime}\right)=r_{1}^{2} \cos \tau$

$\gamma r_{1}^{2} \bar{z}_{2}^{\prime \prime}-\kappa \bar{z}_{1}+\kappa \bar{z}_{2}=\gamma r_{1}^{2} \cos \tau$

and:

$r_{1}^{2} \bar{z}_{1}^{\prime \prime}+(1+\kappa) \bar{z}_{1}-\kappa \bar{z}_{2}=r_{1}^{2} \cos \tau$

$\gamma r_{1}^{2} \bar{z}_{2}^{\prime \prime}-\kappa \bar{z}_{1}+\kappa \bar{z}_{2}+\beta \operatorname{sgn}\left(\bar{z}_{2}^{\prime}\right)=\gamma r_{1}^{2} \cos \tau$

Equations (77) and (78) are representative of the non-dimensional equivalent systems shown in Fig. 13a, b. Following the criteria detailed in Sect. 2.2, it is possible to derive from Eq. (77) the sticking conditions needed for the numerical integration:

$\bar{z}_{1}^{\prime}=0$

$\left|r_{1}^{2} \cos \tau-(1+\kappa) \bar{z}_{1}+\kappa \bar{z}_{2}\right|<\beta$

Equation (79) can be rewritten in terms of $x_{1}$ and $x_{2}$ as:

$\bar{x}_{1}^{\prime}=-\sin \tau$

$\left|\left(1+r_{1}^{2}\right) \cos \tau-(1+\kappa) \bar{z}_{1}+\kappa \bar{z}_{2}\right|<\beta$ 


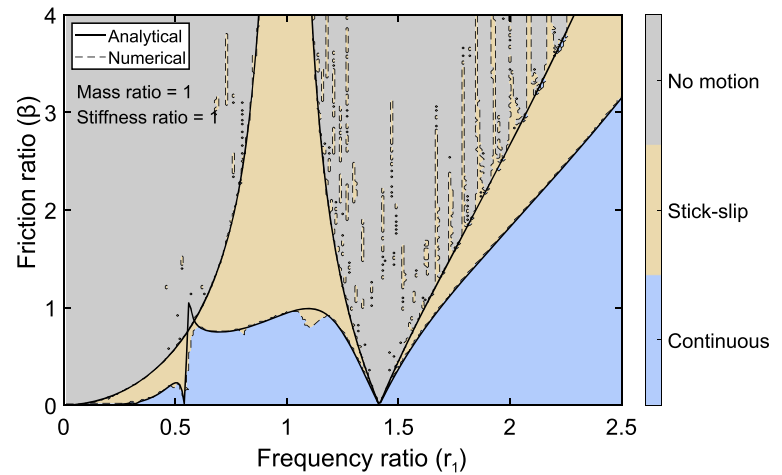

(a) $\gamma=1, \kappa=1$

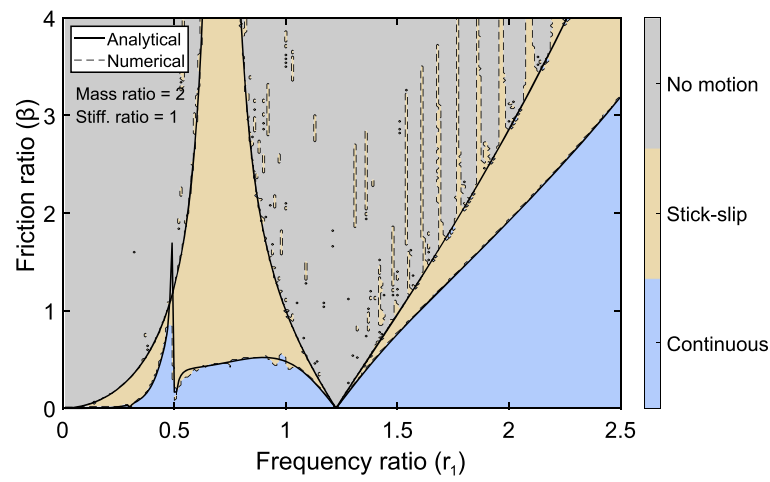

(c) $\gamma=2, \kappa=1$

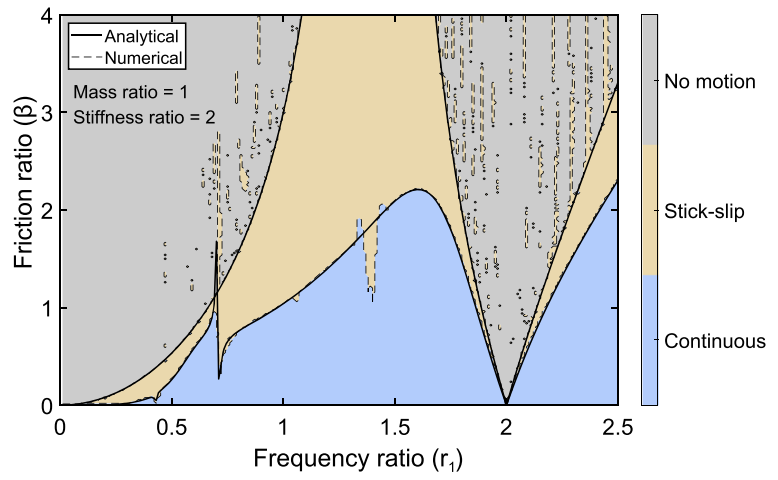

(b) $\gamma=1, \kappa=2$

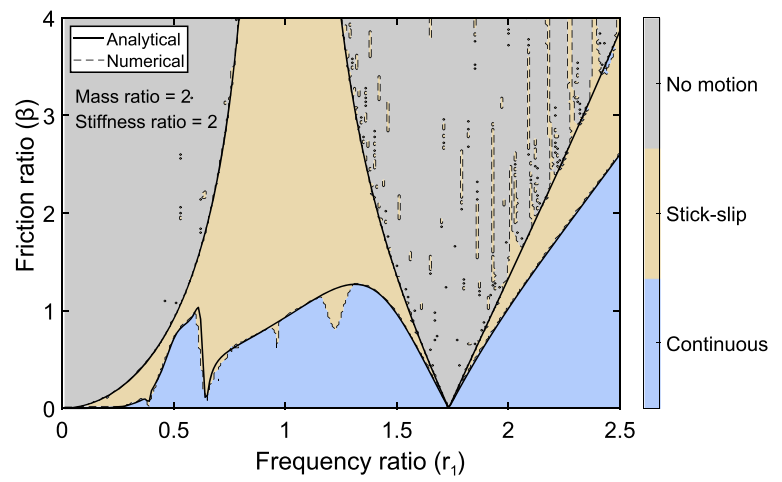

(d) $\gamma=2, \kappa=2$

Fig. 14 Motion regimes of a 2DOF system under harmonic base excitation with a Coulomb contact between the lower mass and a base-fixed wall in the parameter space $r_{1}-\beta$. Coloured regions refer to the numerical results. Each figure corresponds to a different mass ratio $(\gamma)$ and stiffness ratio $(\kappa)$

Similarly, the conditions for the configuration involving a friction contact on the upper mass will be:

$\bar{x}_{2}^{\prime}=-\sin \tau$

$\left|\gamma r_{1}^{2} \cos \tau-\kappa\left(\bar{x}_{2}-\bar{x}_{1}\right)\right|<\beta$

\subsection{Boundary between continuous and stick-slip} regimes for $2 \mathrm{DOF}$ systems

The systems in Fig. 13a, b exhibit the same contact configurations as the systems shown in Fig. 2a, b, which have been referred to when introducing the modal superposition procedure in Sect. 2.4. The only relevant difference between these systems is found in the different load configurations, as the equivalent systems considered here are subjected to dynamic forces directly applied on the masses, rather than to base motion. Let us then write the excitation vector for the current sys- tems as:

$\overline{\mathbf{p}}=\left[\begin{array}{ll}r_{1}^{2} \cos \tau & \gamma r_{1}^{2} \cos \tau\end{array}\right]^{T}$

Substituting Equations (27) and (82) into Eq. (34), it is possible to write the modal force vector as:

$\hat{\mathbf{p}}=r_{1} \cos \tau\left[\frac{1+\gamma \varphi_{1}}{\sqrt{1+\gamma \varphi_{1}^{2}}} \frac{1+\gamma \varphi_{2}}{\sqrt{1+\gamma \varphi_{2}^{2}}}\right]^{T}$

Therefore, considering Eq. (46), it is possible to write the response functions for each mass of the equivalent systems as:

$V_{z_{1}}=\sum_{i=1}^{2} \frac{\left(1+\gamma \varphi_{i}\right) r_{1}^{2}}{1+\gamma \varphi_{i}^{2}}\left(\frac{R_{i}}{r_{1}}\right)^{2} \frac{1}{1-R_{i}^{2}}$ 


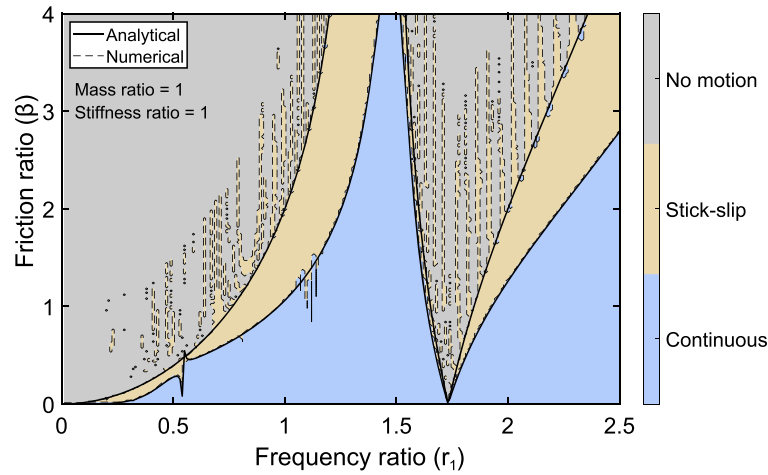

(a) $\gamma=1, \kappa=1$

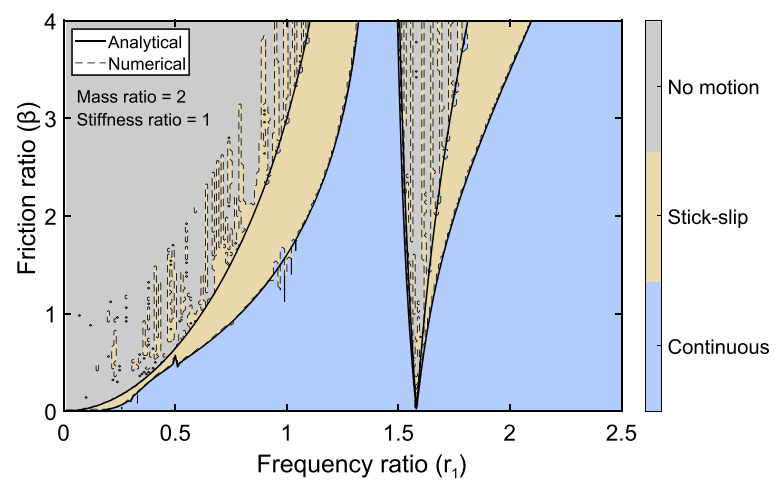

(c) $\gamma=2, \kappa=1$

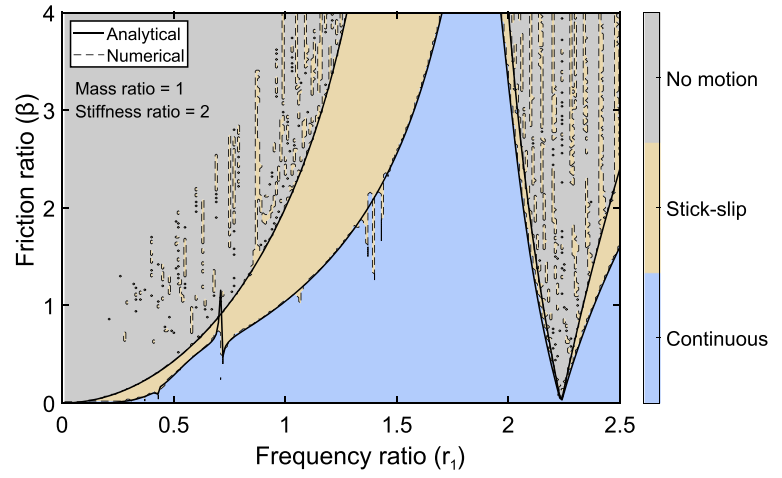

(b) $\gamma=1, \kappa=2$

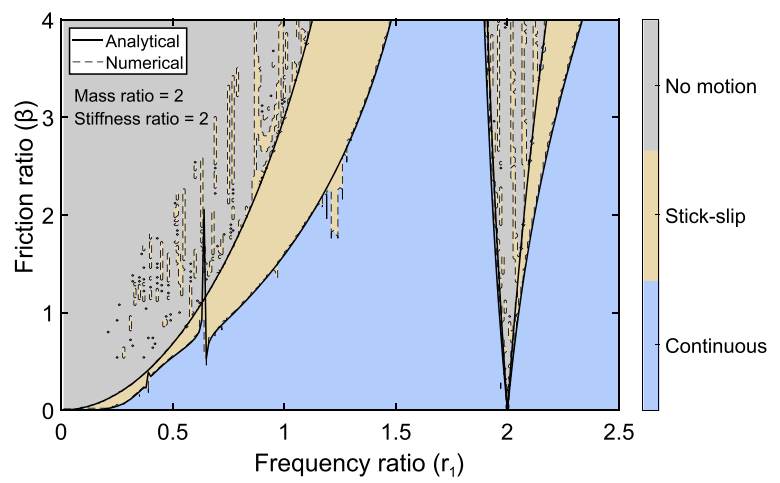

(d) $\gamma=2, \kappa=2$

Fig. 15 Motion regimes of a 2DOF system under harmonic base excitation with a Coulomb contact between the upper mass and a base-fixed wall in the parameter space $r_{1}-\beta$. Coloured regions refer to the numerical results. Each figure corresponds to a different mass ratio $(\gamma)$ and stiffness ratio $(\kappa)$

and:

$V_{z_{2}}=\sum_{i=1}^{2} \frac{\left(1+\gamma \varphi_{i}\right) \varphi_{i} r_{1}^{2}}{1+\gamma \varphi_{i}^{2}}\left(\frac{R_{i}}{r_{1}}\right)^{2} \frac{1}{1-R_{i}^{2}}$

Regarding the damping functions, as the contact configurations and the friction forces are the same considered in Sect. 2, it is possible to write $U_{z_{1}}=U_{1}$ and $U_{z_{2}}=U_{2}$, referring to Eqs. (55) and (58). Finally, the boundaries between continuous and stick-slip regimes are described, respectively, for the two cases, by Eqs. (56) and (59) for $V_{j}=V_{z_{j}}$ and $U_{j}=U_{z_{j}}$.

The analytical boundary between continuous and stick-slip regimes obtained from such a procedure for a 2DOF system with a contact between the lower mass and the base-fixed wall is represented in Fig. 14 for varying stiffness and mass ratios and it shows a good agreement with the numerical results. The curve is split into two parts by an antiresonance, which is further described in Sect. 3.3. At low frequencies, the boundary presents very small values of friction ratio until reaching a first sharp peak in correspondence of the lower natural frequency, while a second smoother peak appears shortly before the antiresonance. In this frequency range, some discrepancies between analytical and numerical results can be observed and they are due to the assumption of $S=1$ (see Sect. 2.3). After the antiresonance, the curve gradually increases to infinity; therefore, it will always be possible to observe a continuous motion between mass and wall by increasing the frequency ratio until a certain threshold value.

The analytical boundary between continuous and stick-slip regimes for the contact configuration involving the upper mass is depicted in Fig. 15. It shows an excellent agreement with the numerical results. The boundary is very similar to the one shown for the previous configuration in Fig. 14, except for a few differ- 
ences, which will be described in more detail in the following subsection.

\subsection{Condition for the presence of a no motion region in 2 DOF systems}

Both Figs. 14 and 15 highlight the presence of regions where no relative motion was observed numerically between the mass and the wall in contact in steadystate conditions. This means that the mass involved in the friction contact is stuck on the base-fixed wall and, therefore, forced to move with the same harmonic motion as the base. As specified in Sects. 2.3 and 2.5, this eventuality occurs when the amplitude of the friction force acting on the mass in contact is larger than the amplitude of the sum of the other forces acting on such a mass when its relative position and velocity are zero.

In order to determine the analytical formulation of the boundary between motion and no-motion regions in the case where the lower mass is in contact, let us consider the non-dimensional equivalent system shown in Fig. 13a. The non-frictional forces acting on such a mass when it is still in $\bar{z}_{1}=0$ are the equivalent exciting load $r_{1}^{2} \cos \tau$, due to the base motion, and the spring force $\kappa \bar{z}_{2}$, due to the motion of the upper mass. Therefore, according to what previously stated, the motion condition will be:

$\left|r_{1}^{2}+\kappa \bar{Z}_{2}\right|>\beta$

If the lower mass is fixed to the wall, the system in Fig. 13a will behave like a SDOF system governed by the equation:

$\gamma r_{1}^{2} \bar{z}_{2}^{\prime \prime}+\kappa \bar{z}_{2}=\gamma r_{1}^{2} \cos \tau$

The amplitude of the response to the excitation $\gamma r_{1}^{2} \cos \tau$ can be determined by imposing $\bar{z}_{2}=\bar{Z}_{2} \cos \tau$ in the above equation and it can be written as:

$\bar{Z}_{2}=\frac{\gamma r_{1}^{2}}{\kappa-\gamma r_{1}^{2}}$
Substituting Eq. (88) into Eq. (86), the final motion condition can be written as:

$\beta<\left|\frac{\kappa(1+\gamma) r_{1}^{2}-\gamma r_{1}^{4}}{\kappa-\gamma r_{1}^{2}}\right|$

The boundary described by Eq. (89) is represented in Fig. 14 and shows a good agreement with numerical results. As shown in the figure, the boundary starts from the origin of the parameter space and increases until reaching an infinite peak at $r_{1}=\sqrt{\kappa / \gamma}$. Further increasing the frequency ratio, the boundary decreases until the already mentioned antiresonance. The frequency ratio of the antiresonance can be determined as a root of the numerator of Eq. (89):

$r_{1}=\sqrt{\frac{\kappa(1+\gamma)}{\gamma}}$

After the antiresonance, the boundary increases to infinity, coherently with what has been observed for the boundary between continuous and stick-slip motions.

The same procedure can be used also for determining the motion condition when the upper mass is in contact with the moving wall. Referring to the nondimensional equivalent system in Fig. 13b, it is possible to observe that, when the upper mass is still, the overall excitation on this mass is given by the sum of the equivalent dynamic load $\gamma r_{1}^{2} \cos \tau$, due to the base motion, and of the spring force $\kappa \bar{z}_{1}$, due to the motion of the lower mass and transmitted by the spring of stiffness $\kappa$. Thus, the motion condition can be written as:

$\left|\gamma r_{1}^{2}+\kappa \bar{Z}_{1}\right|>\beta$

When the upper mass is stuck, the system turns into a SDOF system where the lower mass is connected to a fixed wall by both springs, therefore with an overall stiffness equal to $1+\kappa$. The governing equation will be:

$r_{1}^{2} \bar{z}_{1}^{\prime \prime}+(1+\kappa) \bar{z}_{1}=r_{1}^{2} \cos \tau$

and, therefore, the response amplitude will be:

$\bar{Z}_{1}=\frac{r_{1}^{2}}{1+\kappa-r_{1}^{2}}$ 
Introducing Eq. (93) into Eq. (91), the motion condition becomes:

$\beta<\left|\frac{(\gamma+\gamma \kappa+\kappa) r_{1}^{2}-\gamma r_{1}^{4}}{1+\kappa-r_{1}^{2}}\right|$

Equation (94) describes the boundary between no motion and stick-slip regime in Fig. 15, which shows a very good agreement with the corresponding numerical boundary. The analytical curve shows a similar behaviour to the one described for the previous configuration with two main differences:

- the infinite peak is observed at $r_{1}=\sqrt{1+\kappa}$, which is the root of the denominator of Eq. (94);

- the antiresonance is placed at:

$$
r_{1}=\sqrt{\frac{\gamma+\gamma \kappa+\kappa}{\gamma}}
$$

All the boundaries described in this section are summarised in Table 2.

\subsection{Boundaries for systems with more than two DOFs}

The formulation of the boundaries among motion regimes in the parameter space $r_{1}-\beta$ can be extended to joined base-wall excited systems with a larger number of DOFs, similarly to Sect. 2.6, if only one mass of the system is rubbing against the moving wall.

Also for this contact configuration, a fundamental step is the definition of a system of governing equations for the MDOF system, expressed consistently with the formulation used for 2DOF systems in Sect. 3.1. Let us consider a harmonically excited NDOF system where a friction contact is achieved between the mass $m_{j}$ and the wall. It is possible to write the governing equation for the $j$-th DOF of the system as:

$$
\begin{aligned}
& m_{j} \ddot{x}_{j}-k_{j} x_{j-1}+\left(k_{j}+k_{j+1}\right) x_{j} \\
& \quad-k_{j+1} x_{j+1}+F \operatorname{sgn}\left(\dot{x}_{j}-\dot{y}\right)=0
\end{aligned}
$$

where $x_{j-1}=0$ if $j=1$ and $x_{j+1}=k_{j+1}=0$ if $j=N$. The RHS will be equal to $k_{1} y$ if $j=1$. As proposed in Sect. 3.1, it is convenient to introduce the state variable $z_{j}=x_{j}-y$, so that Eq. (96) can be rewritten as:

$$
\begin{aligned}
& m_{j} \ddot{z}_{j}-k_{j} z_{j-1}+\left(k_{j}+k_{j+1}\right) z_{j} \\
& \quad-k_{j+1} z_{j+1}+F \operatorname{sgn}\left(\dot{z}_{j}\right)=k_{j} r_{j}^{2} y
\end{aligned}
$$

or, in a dimensionless form, as:

$$
\begin{aligned}
& \gamma_{j} r_{1}^{2} \bar{z}_{j}^{\prime \prime}-\kappa_{j} \bar{z}_{j-1}+\left(\kappa_{j}+\kappa_{j+1}\right) \bar{z}_{j} \\
& -\kappa_{j+1} \bar{z}_{j+1}+\beta \operatorname{sgn}\left(\bar{z}_{j}^{\prime}\right)=\gamma_{j} r_{1}^{2} \cos \tau
\end{aligned}
$$

As it can be deduced comparing Eqs. (98) to (65), the introduction of the coordinates $z_{1}, \ldots, z_{N}$ allows the representation of the system as a NDOF system with a ground-fixed contact. Specifically, the mass and stiffness matrices of the system will be the same as in Eqs. (68) and (69). Therefore, all the considerations stated in Sect. 2.6 apply. Particularly, it is worthwhile observing that the joined base-wall excitation produces equivalent dynamic loads equal to $\gamma_{i} r_{1}^{2} \cos \tau$ on all the masses of the system. This means that, unless the mass in contact is placed at bottom $(j=1)$ or the top of the system $(j=N)$, both the lower and the upper undamped subsystems must be taken into account when following the procedure described in Sect. 2.6.

\section{Mass-fixed wall contacts}

This section focuses on the formulation of the boundaries among motion regimes for 2DOF systems where a Coulomb contact is achieved between the two masses, in parallel with a spring. The analytical results found in Sects. 2 and 3 can be extended also to this configuration after finding a variable transformation that allows the formulation of this problem in terms of an equivalent 2DOF system with a ground-fixed contact.

\subsection{Generalities and sticking conditions}

Let us consider a 2DOF system where the masses $m_{1}$ and $m_{2}$ are connected in parallel by a spring of stiffness $k_{2}$ and a Coulomb contact characterised by the friction force $F$. The lower mass $m_{1}$ is connected to the base by a spring of stiffness $k_{1}$; the system is excited by a harmonic base motion $y=Y \cos (\omega t)$ (Fig. 16a). The governing equations of this system can be written as:

$$
\begin{aligned}
& m_{1} \ddot{x}_{1}+\left(k_{1}+k_{2}\right) x_{1} \\
& \quad-k_{2} x_{2}+F \operatorname{sgn}\left(\dot{x}_{1}-\dot{x}_{2}\right)=k_{1} y \\
& m_{2} \ddot{x}_{2}-k_{2} x_{1}+k_{2} x_{2}+F \operatorname{sgn}\left(\dot{x}_{2}-\dot{x}_{1}\right)=0
\end{aligned}
$$

The dynamic behaviour of this system can be analysed by seeking a variables transformation allowing to 
Table 2 Motion regimes of Coulomb damped 2DOF systems with a base-fixed wall configuration for different friction ratio ranges

\begin{tabular}{lll}
\hline Contact & Friction ratio range & Regime \\
\hline$m_{1}$ & $0 \leq \beta<\sqrt{\frac{V_{1}^{2}}{U_{1}^{2}+\frac{1}{r_{1}^{4}}}}$ & Continuous \\
& $\sqrt{\frac{V_{z_{1}}^{2}}{U_{z_{1}}^{2}+\frac{1}{r_{1}^{4}}}} \leq \beta<\left|\frac{\kappa(1+\gamma) r_{1}^{2}-\gamma r_{1}^{4}}{\kappa-\gamma r_{1}^{2}}\right|$ & Stick-slip \\
& $\beta \geq\left|\frac{\kappa(1+\gamma) r_{1}^{2}-\gamma r_{1}^{4}}{\kappa-\gamma r_{1}^{2}}\right|$ & No motion \\
$m_{2}$ & $0 \leq \beta<\sqrt{\frac{V_{2}^{2}}{U_{2}^{2}+\frac{1}{\gamma^{2} r_{1}^{4}}}}$ & Continuous \\
& $\sqrt{\frac{V_{z_{2}}^{2}}{U_{z_{2}}^{2}+\frac{1}{\gamma^{2} r_{1}^{4}}} \leq \beta<\mid \frac{(\gamma+\gamma \kappa+\kappa) r_{1}^{2}-\gamma r_{1}^{4}}{1+\kappa-r_{1}^{2}}} \mid$ & Stick-slip \\
& $\beta \geq\left|\frac{(\gamma+\gamma \kappa+\kappa) r_{1}^{2}-\gamma r_{1}^{4}}{1+\kappa-r_{1}^{2}}\right|$ & \\
\hline
\end{tabular}

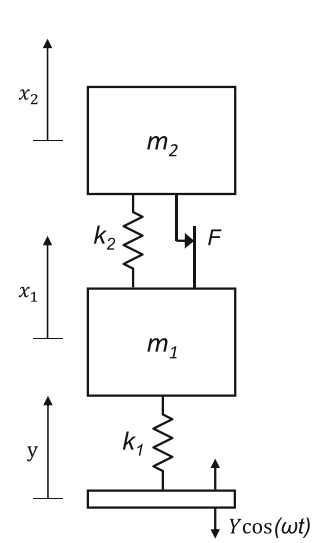

(a)

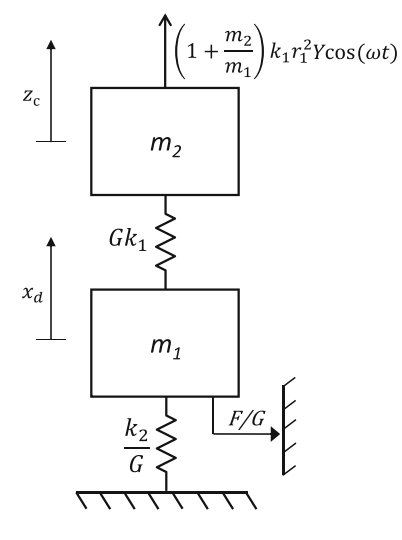

(b)

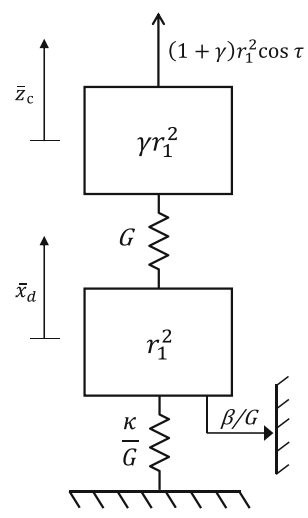

(c)

Fig. 16 2DOF system under harmonic base excitation with a spring and a Coulomb contact in parallel between the masses (a), its equivalent representation as a 2DOF system with a ground-fixed wall contact on the lower mass (b) and the non-dimensional system corresponding to the latter (c)

rewrite Eq. (99) in the same form as Eq. (1). This would allow to extend the results found for ground-fixed wall contacts in Sect. 2.4 to the contact configuration investigated in this section.

The first step is the introduction of the state variable:

$x_{d}=x_{2}-x_{1}$

i.e. the relative displacement between the components in contact. Multiplying Eq. (99a) by $m_{2} / m_{1}$ and subtracting it from Eq. (99b), it is then possible to write:

$$
m_{2} \ddot{x}_{d}+k_{2}\left(1+\frac{m_{2}}{m_{1}}\right) x_{d}
$$

$$
+F\left(1+\frac{m_{2}}{m_{1}}\right) \operatorname{sgn}\left(\dot{x}_{d}\right)=\frac{m_{2}}{m_{1}} k_{1}\left(x_{1}-y\right)
$$

and therefore:

$$
\begin{aligned}
& \frac{m_{1} m_{2}}{m_{1}+m_{2}} \ddot{x}_{d}+k_{2} x_{d} \\
& \quad+F \operatorname{sgn}\left(\dot{x}_{d}\right)=\frac{m_{2}}{m_{1}+m_{2}} k_{1}\left(x_{1}-y\right)
\end{aligned}
$$

Equation (102) partially recalls a result previously described by Den Hartog. In fact, in reference [31], he observes that a system composed by two masses $m_{1}$ and $m_{2}$ connected in parallel by a spring $k$ and a Coulomb contact with friction force $F$, where a harmonic motion $x_{1}=X_{1} \cos (\omega t)$ is imposed on mass $m_{1}$, is equivalent to a SDOF system characterised by: 
- a mass $\frac{m_{1} m_{2}}{m_{1}+m_{2}}$;

- a spring of stiffness $k$;

- a ground-fixed wall contact with friction force F;

- harmonic excitation of amplitude $\frac{m_{2}}{m_{1}} k_{1} x_{1}$.

Therefore, in Den Hartog's system the motion $x_{1}$ is given a priori and not intended as a response, differently from what happens in the system described in this section. Furthermore, Den Hartog's system is not connected to the ground, so it will exhibit only one oscillating mode in addition to a rigid-body motion. Conversely, the system in Fig. 16a is by all means a 2-DOF system, where both $x_{1}$ and $x_{2}$ are unknown, so the problem cannot be reduced to the analysis of an equivalent SDOF system.

Equation (102) also allows the definition of the sticking conditions for this system, useful for the numerical integration approach described in Sect. 4:

$\dot{x}_{d}=0$

$\left|\frac{m_{2}}{m_{1}+m_{2}} k_{1}\left(x_{1}-y\right)-k_{2} x_{d}\right|<F$

or, in terms of $x_{1}$ and $x_{2}$ :

$\dot{x}_{2}=\dot{x}_{1}$

$\mid\left(\frac{m_{2}}{m_{1}+m_{2}} k_{1}+k_{2}\right) x_{1}$

$-k_{2} x_{2}-\frac{m_{2}}{m_{1}+m_{2}} k_{1} y \mid<F$

4.2 Boundary between continuous and stick-slip regimes

Let us introduce the coordinate $x_{c}$ of the centroid of the system:

$x_{c}=\frac{m_{1} x_{1}+m_{2} x_{2}}{m_{1}+m_{2}}$

and consider the sum of Eqs. (99a) and (99b):

$m_{1} \ddot{x}_{1}+m_{2} \ddot{x}_{2}+k_{1}\left(x_{1}-y\right)=0$

This can be rewritten as:

$\left(m_{1}+m_{2}\right) \ddot{x}_{c}=-k_{1}\left(x_{1}-y\right)$
As expected, the motion of the centroid of the system is not influenced by either the friction force or the action of the spring $k_{2}$. By writing $x_{1}$ and $x_{2}$ in terms of the new state coordinates $x_{d}$ and $x_{c}$ :

$x_{1}=x_{c}-\frac{m_{2}}{m_{1}+m_{2}} x_{d}$

$x_{2}=x_{c}+\frac{m_{1}}{m_{1}+m_{2}} x_{d}$

it is possible to remove $x_{1}$ from Eqs. (101) and (107), obtaining the following system of equations:

$$
\begin{gathered}
m_{1} \ddot{x}_{d}+\left(\frac{m_{2}}{m_{1}+m_{2}} k_{1}+\frac{m_{1}+m_{2}}{m_{2}} k_{2}\right) x_{d} \\
-k_{1} x_{c}+\frac{m_{1}+m_{2}}{m_{2}} F \operatorname{sgn}\left(\dot{x}_{d}\right)=-k_{1} y \\
\left(m_{1}+m_{2}\right) \ddot{x}_{c}-\frac{m_{2}}{m_{1}+m_{2}} k_{1} x_{d}+k_{1} x_{c}=k_{1} y
\end{gathered}
$$

Equation (109) provides a useful alternative description of the system in terms of the relative motion between the masses and its centroid. However, although the friction force is now present only in Eq. (109)a, this system does not accomplish the requirement of presenting the same form as Eq. (1); in fact, it does not describe a 2DOF system.

It is possible to further transform Eq. (109) in order to achieve this purpose. First of all, let us introduce the constant:

$G=\frac{m_{2}}{m_{1}+m_{2}}=\frac{\gamma}{1+\gamma}$

in order to keep notation to its minimal. Eq. (109) yields:

$$
\begin{aligned}
& m_{1} \ddot{x}_{d}+\left(G k_{1}+\frac{k_{2}}{G}\right) x_{d} \\
& -k_{1} x_{c}+\frac{F}{G} \operatorname{sgn}\left(\dot{x}_{d}\right)=-k_{1} y \\
& \left(m_{1}+m_{2}\right) \ddot{x}_{c}-G k_{1} x_{d}+k_{1} x_{c}=k_{1} y
\end{aligned}
$$

Consider a new variable $z_{c}$ defined as:

$z_{c}=\frac{1}{G}\left(x_{c}-y\right)$ 


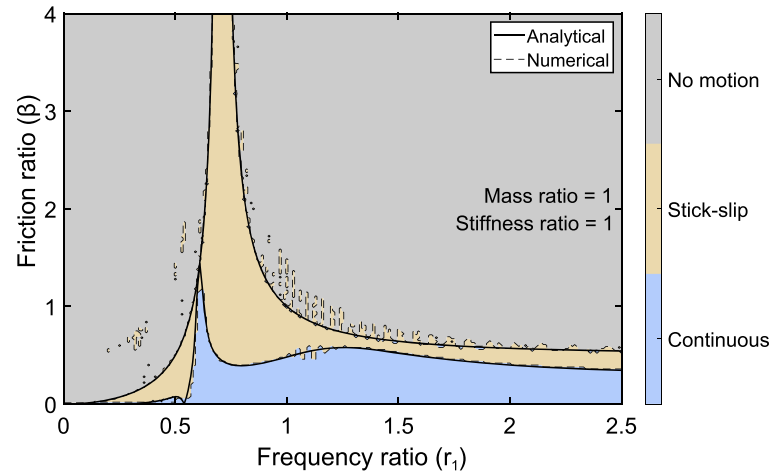

(a) $\gamma=1, \kappa=1$

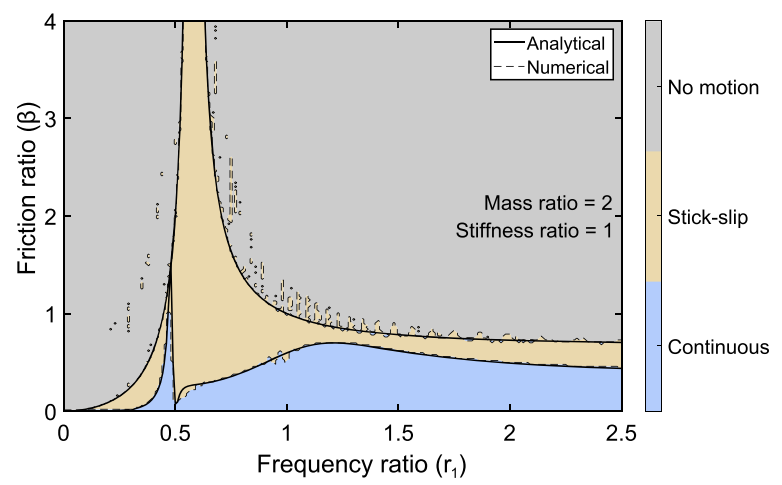

(c) $\gamma=2, \kappa=1$

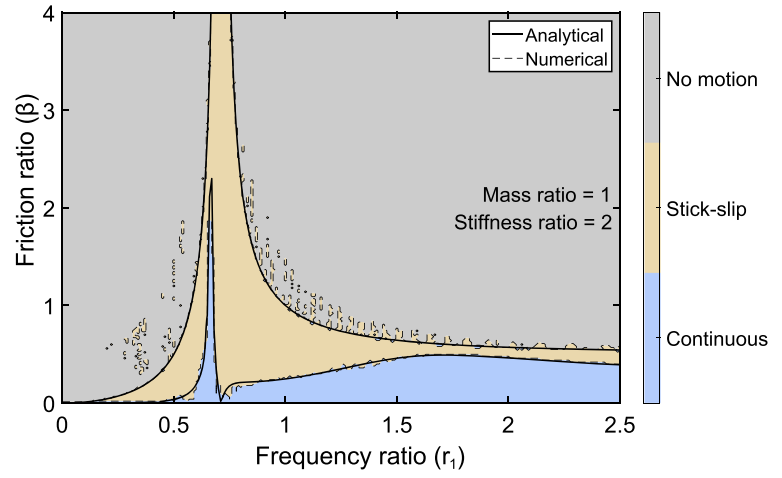

(b) $\gamma=1, \kappa=2$

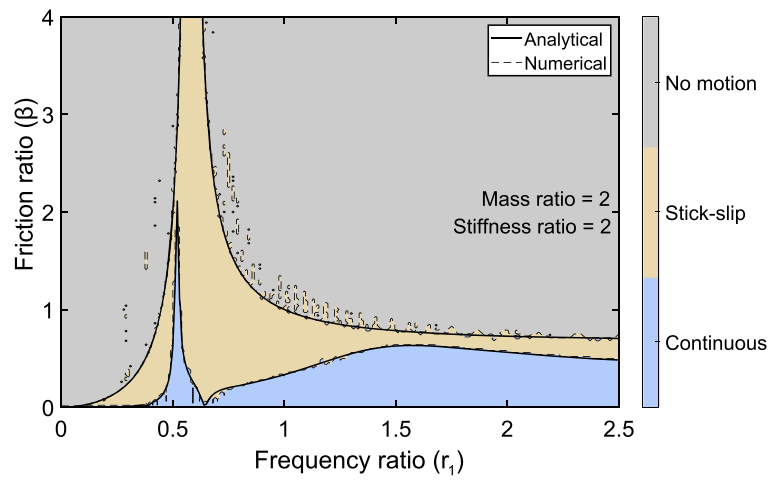

(d) $\gamma=2, \kappa=2$

Fig. 17 Motion regimes of a 2DOF system under harmonic base excitation with a spring and a Coulomb contact in parallel between the masses in the parameter space $r_{1}-\beta$. Coloured regions refer to the numerical results. Each figure corresponds to a different mass ratio $(\gamma)$ and stiffness ratio $(\kappa)$

By substituting Eq. (112) into Eq. (109), the system of equations:

$$
\begin{aligned}
& m_{1} \ddot{x}_{d}+\left(\frac{k_{2}}{G}+G k_{1}\right) x_{d} \\
& -G k_{1} z_{c}+\frac{F}{G} \operatorname{sgn}\left(\dot{x}_{d}\right)=0 \\
& m_{2} \ddot{z}_{c}-G k_{1} x_{d}+G k_{1} z_{c}=\left(1+\frac{m_{2}}{m_{1}}\right) k_{1} r_{1}^{2} y
\end{aligned}
$$

is obtained. Equation (113) describes the equivalent 2DOF system with a ground-fixed wall contact on the lower mass shown in Fig. 16b.

Particularly, this system is excited by a harmonic load $\left(1+m_{2} / m_{1}\right) k_{1} r_{1}^{2} y$ applied on the upper mass; the amplitude of this force depends on the frequency ratio and therefore, for a given system, it will grow when the driving frequency is increased. It is convenient to rewrite Eq. (113) in a non-dimensional form by using the quantities described in Sect. 2.1 and introducing:

$$
\bar{x}_{d}=\frac{x_{d}}{Y} \quad \bar{z}_{c}=\frac{z_{c}}{Y}
$$

Eq. (113) will assume the form:

$$
\begin{aligned}
& r_{1}^{2} \bar{x}_{d}^{\prime \prime}+\left(\frac{\kappa}{G}+G\right) \bar{x}_{d}-G \bar{z}_{c}+\frac{\beta}{G} \operatorname{sgn}\left(\bar{x}_{d}^{\prime}\right)=0 \\
& \gamma r_{1}^{2} \bar{z}_{c}^{\prime \prime}-G \bar{x}_{d}+G \bar{z}_{c}=(1+\gamma) r_{1}^{2} \cos \tau
\end{aligned}
$$

These equations can be seen as the governing equations of an equivalent non-dimensional system, shown in Fig. $16 \mathrm{c}$.

At this point, the procedure introduced in this paper for the analytical determination of the boundaries of the motion regime from the parameters of the system can successfully be applied also to the system considered in this section. The mass matrix of the system will have the same expression as shown in Eq. (19), while the 
stiffness matrix will be:

$$
\overline{\mathbf{K}}=\left[\begin{array}{cc}
\frac{\kappa}{G}+G & -G \\
-G & G
\end{array}\right]
$$

It is possible to evaluate the natural frequencies $\bar{\Omega}_{1,2}$ of the system from Eq. (21) and it can be verified that, despite the different expression of the matrix $\overline{\mathbf{K}}$, they will be the equal to the ones obtained in Eq. (22). The mode shapes can be determined from the general eigenvalue problem indicated in Eq. (18), which yields:

$$
\begin{aligned}
& \left(\frac{\kappa}{G}+G-\bar{\Omega}_{i}^{2} r_{1}^{2}\right) \psi_{1, i}-G \psi_{2, i}=0 \\
& -G \psi_{1, i}+\left(G-\gamma \bar{\Omega}_{i}^{2} r_{1}^{2}\right) \psi_{2, i}=0
\end{aligned}
$$

The ratio between the components of each mode shapes can be determined from either Eq. (117a) or (117b). Considering, for instance, Eq. (117b), the ratio will be:

$\tilde{\varphi}_{i}=\frac{\psi_{i, 2}}{\psi_{i, 1}}=\frac{G}{G-\gamma \bar{\Omega}_{i}^{2} r_{1}^{2}}=\frac{1}{1-(1+\gamma) \bar{\Omega}_{i}^{2} r_{1}^{2}}$

The formulation of $\tilde{\varphi}_{1}$ and $\tilde{\varphi}_{2}$ is different from the one found in Eq. (24), but the mode vectors and the modal matrix will maintain the same form described, respectively, in Eqs. (26) and (27) for $\varphi_{1}=\tilde{\varphi}_{1}$ and $\varphi_{2}=\tilde{\varphi}_{2}$. After introducing the transformation in modal coordinates from Eq. (31), it is necessary to evaluate the modal force, considering that the equivalent load shown in Fig. 16c is applied to the upper mass and has a different amplitude compared to the case studied in Sect. 2. For this system, the applied force vector is:

$\overline{\mathbf{p}}=\left[\begin{array}{ll}0 & (1+\gamma) r_{1}^{2}\end{array}\right]^{T}$

By applying Eq. (34), the corresponding $\hat{\mathbf{p}}$ is found:

$\hat{\mathbf{p}}=(1+\gamma) r_{1} \cos \tau\left[\frac{\tilde{\varphi}_{1}}{\sqrt{1+\gamma \tilde{\varphi}_{1}^{2}}} \frac{\tilde{\varphi}_{2}}{\sqrt{1+\gamma \tilde{\varphi}_{2}^{2}}}\right]^{T}$

Following the same steps done for evaluating $V_{1}$ (in Eq. (46)), the response function $V_{d}$, referred to the rel- ative motion $x_{d}$ (i.e. the lower mass of the equivalent system), can be obtained as:

$V_{d}=\sum_{i=1}^{2} \frac{(1+\gamma) \tilde{\varphi}_{i} r_{1}^{2}}{1+\gamma \tilde{\varphi}_{i}^{2}}\left(\frac{R_{i}}{r_{1}}\right)^{2} \frac{1}{1-R_{i}^{2}}$

Similarly, the damping function can be obtained from Eq. (54). As the equivalent system exhibits the same natural frequencies as the systems studied in Sect. 2 and a formally identical modal matrix, the damping function will have the same form as the function $U_{1}$ described in Eq. (55) for the case of a friction contact applied on the lower mass:

$U_{d}=\sum_{i=1}^{2} \frac{1}{1+\gamma \tilde{\varphi}_{i}^{2}}\left(\frac{R_{i}}{r_{1}}\right)^{2} \frac{\sin \left(\pi / R_{i}\right)}{R_{i}\left[1+\cos \left(\pi / R_{i}\right)\right]}$

In conclusion, the boundary curve between continuous and stick-slip regimes in the $r_{1}-\beta$ parameter space can be written as:

$\beta_{d, \lim }=G \beta_{1, \lim }=\frac{\gamma}{1+\gamma} \sqrt{\frac{V_{d}^{2}}{U_{d}^{2}+\left(\frac{1}{r_{1}^{2}}\right)^{2}}}$

where the expression of $\beta_{1, \text { lim }}$ obtained in Eq. (56) is multiplied by $G$ since the amplitude of the friction force acting in the equivalent system is $\beta / G$.

The boundary curve described by Eq. (123) is shown in Fig. 17 for different values of mass and stiffness ratios and agrees well with the boundary highlighted by numerical results. Starting from low frequencies, continuous motion is possible only for very small friction ratios; a very sharp peak can be observed in correspondence of the first natural frequency of the system. After the peak, the boundary increases reaching a smoother second peak, whose value is always smaller than 1 in the observed cases.

\subsection{Condition for the presence of a no motion region}

In Fig. 17, it is shown clearly that it is not always possible to observe a relative motion between $m_{1}$ and $m_{2}$ as the parameters of system are varied. In the absence of such motion, the system will exhibit a stuck configura- 
tion, reducing to an undamped SDOF system of mass $m_{1}+m_{2}$ and spring $k_{1}$.

The condition for which the relative motion is possible can be described analytically by applying the procedure introduced in Sects. 2.5 and 3.3 to the nondimensional system. Referring to Fig. 16c, it is possible to observe that the only exciting force acting on the lower mass when it is fixed, excluding friction force, is the spring force due to the motion of the upper mass. Being $G$ the stiffness of the upper spring and $\beta / G$ the intensity of the friction force, the motion condition will be:

$$
\left|G \bar{Z}_{c}\right|>\frac{\beta}{G}
$$

which can be rewritten as:

$$
\frac{\gamma^{2}}{(1+\gamma)^{2}}\left|\bar{Z}_{c}\right|>\beta
$$

In order to determine $\bar{Z}_{c}$, it must be considered that, when the lower mass is stuck, the upper spring and the upper mass behave like a SDOF system excited by the equivalent force $(1+\gamma) r_{1}^{2} \cos \tau$ due to the base motion. Thus, the governing equation of this system will be:

$\gamma r_{1}^{2} \bar{z}_{c}^{\prime \prime}+G \bar{z}_{c}=(1+\gamma) r_{1}^{2} \cos \tau$

and the amplitude of the response, obtained by substituting $\bar{z}_{c}=\bar{Z}_{c} \cos \tau$, can be written as:

$\bar{Z}_{c}=\frac{(1+\gamma) r_{1}^{2}}{G-\gamma r_{1}^{2}}$

Substituting Eqs. (110) and (127) into Eq. (125), and after some algebraic manipulations, the motion condition can be finally expressed as:

$\beta<\frac{\gamma r_{1}^{2}}{\left|1-(1+\gamma) r_{1}^{2}\right|}$

This condition describes the boundary between the stick-slip region (in orange) and the no motion region (in grey) in Fig. 17. This boundary starts from the origin of the parameter space and quasi-static motion can be observed only for very small values of the friction ratio. As it can be deduced also from Eq. (128), an infinite
Table 3 Motion regimes of Coulomb damped 2DOF systems with a mass-fixed wall configuration for different friction ratio ranges

\begin{tabular}{ll}
\hline Friction ratio range & Regime \\
\hline $0 \leq \beta<\frac{\gamma}{1+\gamma} \sqrt{\frac{V_{d}^{2}}{U_{d}^{2}+\frac{1}{r_{1}^{4}}}}$ & Continuous \\
$\frac{\gamma}{1+\gamma} \sqrt{\frac{V_{d}^{2}}{U_{d}^{2}+\frac{1}{r_{1}^{4}}}} \leq \beta<\frac{\gamma r_{1}^{2}}{\left|1-(1+\gamma) r_{1}^{2}\right|}$ & Stick-slip \\
$\beta \geq \frac{\gamma r_{1}^{2}}{\left|1-(1+\gamma) r_{1}^{2}\right|}$ & No motion \\
\hline
\end{tabular}

peak is reached at:

$r_{1}=\sqrt{\frac{1}{1+\gamma}}$

After the peak, the boundary decreases until reaching an asymptotic value given by:

$\beta_{\infty}=\frac{\gamma}{1+\gamma}$

when $r_{1} \rightarrow+\infty$. Finally, it is interesting to observe that the motion condition is independent of the stiffness ratio.

The motion regime scenario described in this section is summarised in Table 3.

\section{Numerical approach}

In the previous sections, numerical results have been used for validating the analytical expressions of the bounds among motion regimes in MDOF systems with different configurations of Coulomb contacts. This section focuses on the description of the numerical methods used for this purpose.

The numerical integration of the governing equations of the MDOF systems analysed in this paper can be performed using standard numerical solvers as long as the solution is continuous; however, particular care must be taken when sticking phases appear in the motion. In fact, in stick-slip regime, the transitions between sliding and sticking phases cause sudden variations in the solution, which cannot be easily dealt with 
by most numerical methods. Stiff solvers are usually implemented in order to address this particular numerical problem (see, e.g. [32]).

Nevertheless, in reference [33], it was shown that better performances in terms of accuracy and computational cost can be achieved for stick-slip motion in SDOF systems if a standard nonstiff solver is used for the integration during the sliding stages and explicit conditions are set a priori to account for the transitions between the sliding and the sticking regimes. This approach has been extended in this paper to account for MDOF systems and is detailed below, for simplicity, in the $2 \mathrm{DOF}$ case. However, the same procedure can also be used to account for systems with a larger number of DOFs.

In the presence of either ground-fixed or base-fixed contacts, only the mass in contact will be stuck on the wall. In fact, the remaining mass will keep oscillating and, therefore, also during the sticking phases, its motion will not be known a priori. The integration process can be summarised as follows.

- During the sliding phases, both masses are oscillating continuously and the solution is nonstiff, so the governing equations are integrated by using a variable-step Runge-Kutta $(4,5)$ method, implemented in the Matlab function ode45 [34].

- The integration is stopped when the relative velocity between mass and wall, i.e. the argument of the $s g n$ function, is equal to zero. If also the second sticking condition, defined in Eqs.(11b) and (12b) for ground-fixed contacts and in Eqs.(80b) and (81b) for base-fixed contacts, is verified, a sticking phase will start; otherwise, a further sliding phase will follow.

- During the sticking phases, the mass in contact will move jointly to the wall, so its displacement $\bar{x}_{s}$ and its velocity $\bar{x}_{s}^{\prime}$ are imposed. Specifically, if the stop occurs at time $\tau_{0}$ and at the position $\bar{x}_{s_{0}}$, the imposed values will be:

$$
\bar{x}_{s}=\bar{x}_{s_{0}} \quad \bar{x}_{s}^{\prime}=0
$$

for a stuck ground-fixed contact and:

$$
\bar{x}_{s}=\bar{x}_{s_{0}}-\cos \tau_{0}+\cos \tau \quad \bar{x}_{s}^{\prime}=-\sin \tau
$$

for a stuck base-fixed contact.
- At the same time, numerical integration is performed for the mass not in contact, whose motion represents the only unconstrained degree of freedom of the system at this stage. Therefore, the dynamic behaviour of the system will be described by a single equation. For instance, if the case of a ground-fixed contact on the lower mass is considered, the governing equation is obtained by posing $\bar{x}_{1}=\bar{x}_{s}$. Substituting Eq. (131) into Eq. (4b), it can be written as:

$$
\gamma r_{1}^{2} \bar{x}_{2}^{\prime \prime}+\kappa \bar{x}_{2}=\kappa \bar{x}_{s_{0}}
$$

All the other configurations analysed in this paper can be dealt with similarly.

- The sticking phase will be stopped when the second sticking condition is no longer verified, i.e. when the resultant dynamic load overcomes the friction force.

In mass-fixed contacts, the sticking occurs between the masses so this case needs to be addressed differently. As specified in Sect. 4.3, when the sticking occurs, i.e. when the sticking conditions expressed in Eq. (104), the system will transition to a stuck configuration, behaving as an undamped SDOF system of mass $m_{1}+m_{2}$ and stiffness $k_{1}$. Indicating with $\bar{x}_{d_{0}}$ the relative displacement between the two masses when the stop occurs and referring to Eq. (109b), it is possible to write the only governing equation needed for describing the dynamic behaviour of the system in a stuck configuration as:

$(1+\gamma) r_{1}^{2} \bar{x}_{c}^{\prime \prime}+\bar{x}_{c}=\cos \tau+G \bar{x}_{d_{0}}$

The position of the two masses during this stage will be determined, from Eq. (108), as:

$\bar{x}_{1}=\bar{x}_{c}-G \bar{x}_{d_{0}}$

$\bar{x}_{2}=\bar{x}_{c}+(1-G) \bar{x}_{d_{0}}$

In this paper, each integration, for varying $r_{1}, \beta, \gamma$ and $\kappa$ parameters, has been performed for 100 cycles of base motion, aiming to determine the motion regime in steady-state conditions. For almost all the configuration of these parameters, no change of regime could be observed with longer durations. A few exceptions are described in the literature, regarding transitions 
between continuous and stick-slip regimes after a considerable number of motion cycles (see, e.g. reference [6]), but they were not considered relevant within the purposes of this numerical analysis, as limited to a few particular sets of parameters. As already mentioned in Sect. 2.5, residual motion in the friction contacts was sometimes observed after 100 excitation cycles above the boundary between stick-slip and motion regimes; this resulted in the small spikes observable in most of the graphical representations of the parameter space $r_{1}-\beta$ presented in this paper. Nevertheless, the amplitude of such residual motions was found to be negligible in most cases. During the integration process, the absolute and relative tolerances were set, respectively, to $10^{-6}$ and $10^{-12}$.

\section{Concluding remarks}

The analytical boundaries of motion regimes for three types of MDOF systems with a Coulomb friction contact have been investigated. Specifically, the boundaries among regions of: (1) continuous motion; (2) stick-slip motion; (3) no motion have been investigated in a nondimensional parameter space in terms of the frequency ratio and the friction ratio.

The boundaries were evaluated in closed-form and validated numerically for $2 \mathrm{DOF}$ systems with a (i) ground-fixed, (ii) base-fixed and (iii) mass-fixed wall contact. A procedure for extending these results to systems with more than two DOFs was also proposed for the cases (i) and (ii), with a further numerical validation for the case of a 5DOF system with a ground-fixed wall contact.

The approach for the definition of the boundary between continuous (non-sticking) and stick-slip regimes was directly achieved for 2DOF systems with a fixed-wall Coulomb contact on either the upper or the lower mass by considering the superposition of the modal behaviour and applying Den Hartog's approach [2] for determining the response of each mode. Differently, the non-sticking conditions for 2DOF systems presenting the contact configurations (ii) and (iii) were obtained by reducing these systems to equivalent configurations with a ground-fixed wall contact. This was achieved by introducing appropriate variable transformations in the governing equation of such systems.

An ad hoc procedure was introduced for the determination of the boundary between motion and no motion regions, based on the principle that sliding will occur in a friction joint only if the overall dynamic load applied on the components in contact has a larger amplitude than the friction force.

An excellent agreement was observed when comparing the analytical and the numerical boundaries for 2DOF systems in all the cases analysed. The investigation of the parameter space highlighted how the shape and the extension of regions associated with the three motion regimes change significantly when different mass and stiffness ratios, wall motions or masses in contact are considered. It was observed that, for particular configurations and parameters, the boundary curves can be very close or overlap, generating regions where different motion regimes can be verified if small variations are introduced in either the friction or the exciting forces. This dynamic behaviour is usually unsuitable for structural design. Finally, it was shown that the boundary between motion and no motion regions is independent of: (i) the mass ratio for a ground-fixed wall contact and (ii) the stiffness ratio when the contact occurs between the two masses.

Overall, the presented results give information relevant to the design and the analysis of friction joints in engineering structures. Current work on this topic is focusing on the analysis of the dynamic response features of MDOF systems with a Coulomb contact. Moreover, future work will focus on (i) the determination of motion regimes for discrete systems with more than one friction contact and (ii) the extension of this approach to continuous multi-modal structures.

Acknowledgements Luca Marino thanks the EPSRC and Rolls-Royce for an industrial CASE postgraduate scholarship. Alice Cicirello gratefully acknowledges Rolls-Royce plc and the EPSRC for the support under the Prosperity Partnership Grant Cornerstone: Mechanical Engineering Science to Enable Aero Propulsion Futures, Grant Ref: EP/R004951/1. The authors would also like to thank the anonymous reviewers for their constructive comments.

\section{Compliance with ethical standards}

Conflicts of interest The authors declare that they have no conflict of interest.

Open Access This article is licensed under a Creative Commons Attribution 4.0 International License, which permits use, sharing, adaptation, distribution and reproduction in any medium or format, as long as you give appropriate credit to the original author(s) and the source, provide a link to the Creative Commons licence, and indicate if changes were made. The images or other third party material in this article are included in the article's 
Creative Commons licence, unless indicated otherwise in a credit line to the material. If material is not included in the article's Creative Commons licence and your intended use is not permitted by statutory regulation or exceeds the permitted use, you will need to obtain permission directly from the copyright holder. To view a copy of this licence, visit http://creativecommons.org/licenses/ by/4.0/.

\section{References}

1. Berman, A.D., Ducker, W.A., Israelachvili, J.N.: Experimental and theoretical investigations of stick-slip friction mechanisms. In: Persson, B.N.J., Tosatti E.: Physics of Sliding Friction. NATO ASI Series (Series E: Applied Sciences), vol. 311. Springer, Dordrecht (1996)

2. Den Hartog, J.P.: Forced vibrations with combined viscous and Coulomb friction. Trans. Am. Soc. Mech. Eng. 53, 107$115(1931)$

3. Hundal, M.S.: Response of a base excited system with Coulomb and viscous friction. J. Sound Vib. 64, 371-378 (1979)

4. Shaw, S.W.: On the dynamic response of a system with dry friction. J. Sound Vib. 108, 305-325 (1986)

5. Hong, H.-K., Liu, C.-S.: Non-sticking oscillation formulae for Coulomb friction under harmonic loading. J. Sound Vib. 244, 883-898 (2001)

6. Csernak, G., Stepan, G., Shaw, S.W.: Sub-harmonic resonant solutions of a harmonically excited dry friction oscillator. Nonlinear Dyn. 50, 93-109 (2007)

7. Hong, H.-K., Liu, C.-S.: Coulomb friction oscillator: modelling and responses to harmonic loads and base excitations. J. Sound Vib. 229, 1171-1192 (2000)

8. Papangelo, A., Ciavarella, M.: On the limits of quasistatic analysis for a simple Coulomb frictional oscillator in response to harmonic loads. J. Sound Vib. 339, 280-289 (2014)

9. Riddoch, D.J., Cicirello, A., Hills, D.A.: Response of a massspring system subject to Coulomb damping and harmonic base excitation. Int. J. Solids Struct. 193, 527-534 (2020)

10. Marino, L., Cicirello, A., Hills, D.A.: Displacement transmissibility of a Coulomb friction oscillator subject to joined base-wall motion. Nonlinear Dyn. 98, 2595-2612 (2019)

11. Yu, S.D., Wen, S.C.: Vibration analysis of multiple degrees of freedom mechanical system with dry friction. J. Mech. Eng. Sci. 227, 1505-1514 (2013)

12. Meijaard, J.P.: Efficient numerical integration of the equations of motion of non-smooth mechanical systems. Z. Angew. Math. Mech. 77, 419-427 (1997)

13. De la Cruz, S.T., Lopez-Almansa, F., Bozzo, L., Pujades, L.: Numerical simulation of structures equipped with friction energy dissipation devices subjected to seismic load. Trans. Built Env. 57, 261-270 (2001)

14. Toufine, A., Barrau, J.J., Berthillier, M.: Dynamic study of a simplified mechanical system with presence of dry friction. J. Sound Vib. 225, 95-109 (1999)

15. Griffin, J.H.: Friction damping of resonant stresses in gas turbine engine airfoils. ASME J. Eng. Power 102, 329-333 (1980)
16. Ostachowicz, W.: The harmonic balance method for determining the vibration parameters in damped dynamic systems. J. Sound Vib. 131, 465-473 (1989)

17. Dowell, E.H., Schwartz, H.B.: Forced response of a cantilever beam with a dry friction damper attached (I Theory). J. Sound Vib. 91, 255-267 (1983)

18. Dowell, E.H., Schwartz, H.B.: Forced response of a cantilever beam with a dry friction damper attached (II Experiment). J. Sound Vib. 91, 269-291 (1983)

19. Ferri, A.A.: The dynamics of dry friction damped systems. A dissertation presented to the Faculty of Princeton University in Candidacy for the Degree of Doctor of Philosophy (1985)

20. Ferri, A.A., Dowell, E.H.: Frequency domain solutions to multi-degree-of-freedom, dry friction damped systems. J. Sound Vib. 124, 207-224 (1988)

21. Liu, T., Zhang, D., Xie, Y.: A nonlinear vibration analysis of forced response for a bladed-disk with dry friction dampers. J. Low Freq. Noise V. A. 38, 1522-1539 (2019)

22. Rizvi, A., Smith, C.W., Rajasekaran, R., Evans, K.E.: Dynamics of dry friction damping in gas turbines: literature survey. J. Vib. Control 22, 296-305 (2014)

23. Yeh, G.C.K.: Forced vibrations of a two-degree-of-freedom system with combined Coulomb and viscous damping. J. Acoust. Soc. Am. 39, 14-24 (1966)

24. Pascal, M.: Dynamics of coupled oscillators excited by dry friction. ASME J. Comput. Nonlinear Dyn. 3, 20-26 (2008)

25. Pascal, M.: New limit cycles of dry friction oscillators under harmonic load. Nonlinear Dyn. 70, 1435-1443 (2012)

26. Pascal, M.: Sticking and nonsticking orbits for a two-degreeof-freedom oscillator excited by dry friction and harmonic loading. Nonlinear Dyn. 77, 267-276 (2014)

27. Fang, H.-B., Xu, J.: Dynamics of a three-module vibrationdriven system with non-symmetric Coulomb's dry friction. Multibody Syst. Dyn. 27, 455-485 (2012)

28. Ricciardelli, F., Vickery, B.J.: Tuned vibration absorbers with dry friction damping. Earthquake Eng. Struct. Dyn. 28, 707-723 (1999)

29. Inman, D.J.: Engineering Vibration, 4th edn. Pearson Education, International Edition (2014)

30. Craig, R.R., Kurdila, A.J.: Fundamentals of Structural Dynamics. Wiley, Hoboken (2006)

31. Den Hartog, J.P.: Forced vibrations with combined viscous and Coulomb damping. Phil. Mag. VII 9(59), 801-817 (1930)

32. Byrne, G.D., Hindmarsh, A.C.: Stiff ODE solvers: a review of current and coming attractions. J. Comput. Phys. 70, 1-62 (1987)

33. Marino, L., Cicirello, A.: Experimental investigation of a single-degree-of-freedom system with Coulomb friction. Nonlinear Dyn. 99, 1781-1799 (2020)

34. MATLAB, Version 9.3.0.713579 (R2017b). The MathWorks Inc., Natick, MA (2017)

Publisher's Note Springer Nature remains neutral with regard to jurisdictional claims in published maps and institutional affiliations. 